\title{
Hamiltonian unknottedness of certain monotone Lagrangian tori in $S^{2} \times S^{2}$
}

\author{
K. Cieliebak and M. Schwingenheuer
}

\begin{abstract}
We prove that a monotone Lagrangian torus in $S^{2} \times S^{2}$ which suitably sits in a symplectic fibration with two sections in its complement is Hamiltonian isotopic to the Clifford torus.
\end{abstract}

\section{Introduction}

The classification of Lagrangian submanifolds in symplectic manifolds up to Lagrangian or Hamiltonian isotopy is an intriguing problem of symplectic topology. While there are many tools for distinguishing Lagrangian submanifolds, actual classification results have been very rare and restricted to special manifolds in dimension 4 . The first circle of results concerns Lagrangian 2-spheres, in which case the two notions of isotopy coincide: There is a unique 2-sphere up to Hamiltonian isotopy in $S^{2} \times S^{2}$ (Hind [11]), in $T^{*} S^{2}$ and some other Stein surfaces (Hind [12]), and in certain del Pezzo surfaces (Evans [7]). The second circle of results is contained in A. Ivrii's PhD thesis 13: It asserts the uniqueness up to Lagrangian isotopy of Lagrangian tori in $\mathbb{R}^{4}, S^{2} \times S^{2}, \mathbb{C P}^{2}$, and $T^{*} \mathbb{T}^{2}$.

Motivated by Ivrii's thesis, we address in this paper the question of Hamiltonian unknottedness of monotone Lagrangian tori in $S^{2} \times S^{2}$. Recall that a Lagrangian torus is called monotone if its Maslov class is a positive multiple of its symplectic area class on relative $\pi_{2}$. The product of the equators in each $S^{2}$-factor in $S^{2} \times S^{2}$ is called the standard Lagrangian torus $L_{\text {std }}$, or the Clifford torus. This torus is monotone for the standard split symplectic form $\omega_{\text {std }}=\sigma_{\text {std }} \oplus \sigma_{\text {std }}$. There have been many constructions of monotone Lagrangian tori in $\left(S^{2} \times S^{2}, \omega_{\text {std }}\right)$ that are not Hamiltoniian isotopic to $L_{\text {std }}$ due to Eliashberg-Polterovich [5], Chekanov-Schlenk 4, Entov-Polterovich 6, Biran-Cornea 3], Fukaya-OhOhta-Ono [8], and Albers-Frauenfelder [1]. Since they were all recently shown to be Hamiltonian isotopic to each other 9, 16, we will collectively refer to them as the Chekanov-Schlenk torus $L_{C S}$.

The following definition is implicit in Ivrii's thesis. Let us call a monotone Lagrangian torus $L$ in $\left(S^{2} \times S^{2}, \omega_{\text {std }}\right)$ fibered if there exists a foliation $\mathcal{F}$ of $S^{2} \times S^{2}$ by symplectic 2 -spheres in the homology class $\left[p t \times S^{2}\right]$ and a symplectic submanifold $\Sigma$ in class $\left[S^{2} \times p t\right]$ with the following properties: 
- $\Sigma$ is transverse to the leaves of $\mathcal{F}$ and is disjoint from $L$;

- the leaves of $\mathcal{F}$ intersect $L$ in circles (or not at all).

Note that each leaf of $\mathcal{F}$ which intersects the torus $L$ is cut by $L$ into two closed disks glued along $L$. The disks that intersect $\Sigma$ together form a solid torus $T$ with $\partial T=L$.

The first part of Ivrii's thesis now asserts that any monotone Lagrangian torus in $S^{2} \times S^{2}$ is fibered. In this paper, we prove1

Theorem 1.1 (Main Theorem). Let $L \subset\left(S^{2} \times S^{2}, \omega_{\text {std }}\right)$ be a monotone Lagrangian torus which is fibered by $\mathcal{F}$ and $\Sigma$. Assume in addition, that there exists a second symplectic submanifold $\Sigma^{\prime}$ in homology class $\left[S^{2} \times p t\right]$ which is transverse to the leaves of $\mathcal{F}$, and which is disjoint from $\Sigma$ and $T$. Then $L$ is Hamiltonian isotopic to the standard torus $L_{\mathrm{std}}$.

This means that the Chekanov-Schlenk torus $L_{C S}$, or any other exotic monotone Lagrangian torus, cannot possess the additional section $\Sigma^{\prime}$ required in Theorem 1.1. It also suggests that the classification of monotone Lagrangian tori in $\left(S^{2} \times S^{2}, \omega_{\text {std }}\right)$ up to Hamiltonian isotopy may come within reach once we understand better the role of the second section $\Sigma^{\prime}$.

Remark 1.2. It is tempting to conjecture that the Clifford torus and the ChekanovSchlenk torus are the only monotone Lagrangian tori in $\left(S^{2} \times S^{2}, \omega_{\text {std }}\right)$ up to Hamiltonian isotopy. We point out that R. Vianna [19, 20] recently constructed infinitely many pairwise Hamiltonian non-isotopic Lagrangian tori in $\mathbb{C} P^{2}$, and conjectured the existence of a monotone Lagrangian tori in $\left(S^{2} \times S^{2}, \omega_{\text {std }}\right)$ which is not Hamiltonian isotopic to either $L_{\text {std }}$ or $L_{C S}$.

Let us now outline the proof of the main theorem, and in particular explain where the second section is needed. By a relative symplectic fibration on $S^{2} \times S^{2}$ we will mean a quintuple

$$
\mathfrak{S}=\left(\mathcal{F}, \omega, L, \Sigma, \Sigma^{\prime}\right),
$$

as in Theorem 1.1 only with the standard form $\omega_{\text {std }}$ replaced by any symplectic form $\omega$ cohomologous to $\omega_{\text {std }}$. We will prove (Corollary 4.13) that for every symplectic fibration $\mathfrak{S}$ with $\omega=\omega_{\text {std }}$ there exists a homotopy of relative symplectic fibrations $\mathfrak{S}_{t}=\left(\mathcal{F}_{t}, \omega_{\text {std }}, L_{t}, \Sigma_{t}, \Sigma_{t}^{\prime}\right)$ with fixed symplectic form $\omega_{\text {std }}$ such that $\mathfrak{S}_{0}=\mathfrak{S}$ and $\mathfrak{S}_{1}=\mathfrak{S}_{\text {std }}:=\left(\mathcal{F}_{\text {std }}, \omega_{\text {std }}, L_{\text {std }}, S_{0}, S_{\infty}\right)$, where $\mathcal{F}_{\text {std }}$ denotes the standard foliation with leaves $\{z\} \times S^{2}$ and $S_{0}=S^{2} \times\{S\}, S_{\infty}=S^{2} \times\{N\}$ are the standard sections at the south and north pole. Then $L_{t}$ is an isotopy of monotone Lagrangian tori with respect to $\omega_{\text {std }}$ from $L$ to $L_{\text {std }}$, which is Hamiltonian by Banyaga's isotopy extension theorem.

A relative symplectic fibration $\mathfrak{S}$ gives rise to a symplectic fibration $p: S^{2} \times$ $S^{2} \rightarrow \Sigma$ by sending each leaf of $\mathcal{F}$ to its intersection point with $\Sigma$. It determines a symplectic connection whose horizontal subspaces are the symplectic

\footnotetext{
1 Let us emphasize that, while inspired by it, the results in this paper are independent of Ivrii's thesis (which is, unfortunately, neither published nor otherwise accessible).
} 
orthogonal complements to the fibres. Parallel transport along closed paths $\gamma:[0,1] \rightarrow \Sigma$ gives holonomy maps which are symplectomorphisms of the fibre $p^{-1}(\gamma(0))$ and measure the non-integrability of the horizontal distribution. It is not hard to show that a symplectic fibration $\mathfrak{S}$ with trivial holonomy around all loops is diffeomorphic to $\mathfrak{S}_{\text {std }}$, and a theorem of Gromov implies that they are actually homotopic with fixed symplectic form if they both have symplectic form $\omega_{\text {std }}$.

Thus, most of the work will go into deforming a given relative symplectic fibration $\mathfrak{S}$ to one with trivial holonomy. After pulling back $\mathfrak{S}$ by a diffeomorphism, we may assume that $\left(\mathcal{F}, L, \Sigma, \Sigma^{\prime}\right)=\left(\mathcal{F}_{\text {std }}, L_{\text {std }}, S_{0}, S_{\infty}\right)$ (but the symplectic form $\omega$ is nonstandard). In the first step, which takes up Section 3, we make the holonomy trivial near the two sections and near the fibres over the line of longitude through Greenwich in the base, see Figure 1.

Fibre Base

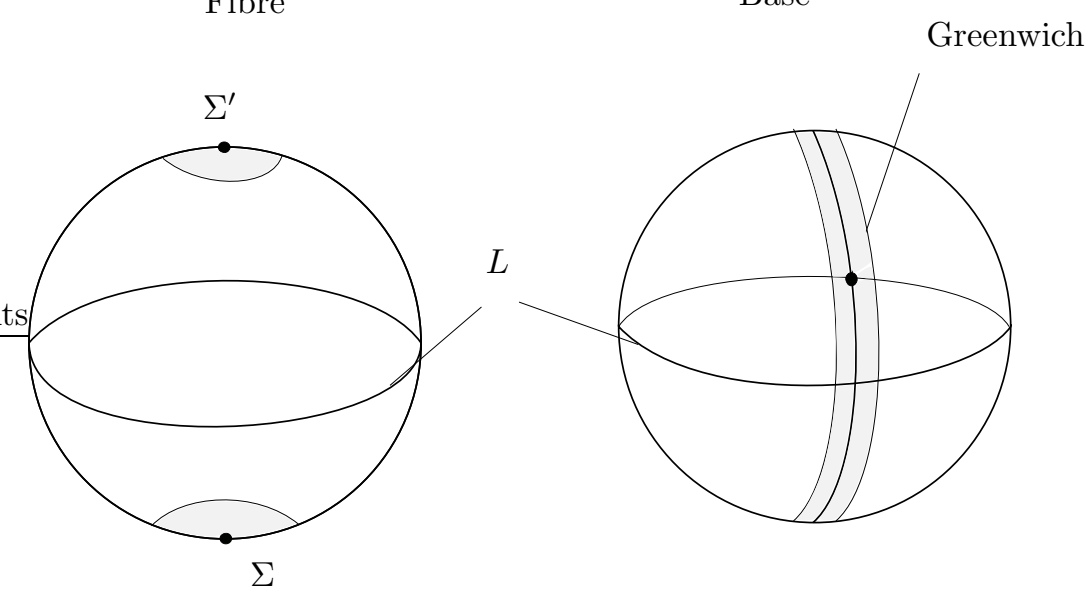

Figure 1: Where the holonomy is trivial after the first step

In the second step, which takes up most of Section 4, we kill the holonomy along all circles of latitude $C^{\lambda}$. For this, let $(\lambda, \mu)$ be spherical coordinates on $S^{2}$, where $\lambda$ denotes the latitude and $\mu$ denotes the longitude. After the first step, the holonomy maps $\phi^{\lambda}$ along $C^{\lambda}$ give a loop in $\operatorname{Symp}\left(A, \partial A, \sigma_{\text {std }}\right)$, the group of symplectomorphisms of the annulus (the sphere minus two polar caps) which equal the identity near the boundary. Since the fundamental group of $\operatorname{Symp}\left(A, \partial A, \sigma_{\text {std }}\right)$ vanishes, we can contract the loop of inverses $\psi^{\lambda}=\left(\phi^{\lambda}\right)^{-1}$ and obtain a family of Hamiltonians $H_{\mu}^{\lambda}$ which generates the contraction. The closed 2-form

$$
\Omega_{H}=\omega+d\left(H_{\mu}^{\lambda} d \mu\right)
$$

then defines a symplectic connection with trivial holonomy around all $C^{\lambda}$. However, $\Omega_{H}$ need not be symplectic if $\frac{\partial H_{\mu}^{\lambda}}{\partial \lambda}$ is large. This can be remedied by the inflation procedure due to Lalonde and McDuff [14. In this procedure, the symplectic form $\omega$ is deformed along a fibre and a section (and $H$ suitably rescaled) 
in order to make $\Omega_{H}$ symplectic. However, this process will in general destroy monotonicity of $L_{\text {std }}$. In order to keep the Lagrangian torus monotone, we perform the inflation procedure along a fibre and the two sections $S_{0}, S_{\infty}$ in a symmetric way. It is at this point of the proof that we need the existence of a second symplectic section.

Once the holonomy along circles of latitude is trivial, in the third and final step (at the end of Section 4) we deform the symplectic form to the standard form. This finishes the outline of the proof.

Remark 1.3. The idea to apply the results of Ivrii's thesis to the Hamiltonian classification of monotone tori in $S^{2} \times S^{2}$ originated in 2003 in the first author's discussions with Y. Eliashberg. However, at the time we did not realize the necessity of a second symplectic section and were puzzled by the apparent contradiction between this result and the existence of an exotic monotone torus in $S^{2} \times S^{2}$. This discrepancy was resolved in the second author's $\mathrm{PhD}$ thesis [18, of which this article is a shortened version.

\section{Acknowledgement}

We thank Y. Eliashberg for many fruitful discussions and his continued interest in this work.

\section{Relative symplectic fibrations}

\subsection{Symplectic connections and their holonomy}

Consider a smooth fibration (by which we mean a fibre bundle) $p: M \rightarrow B$ and a closed 2 -form $\omega$ on $M$ whose restriction to each fibre $p^{-1}(b)$ is nondegenerate. We will refer to $\omega$ as a symplectic connection on $M 2$ From the next subsection on we will assume $\omega$ to be symplectic, but for now this is not needed.

Parallel transport. Since $\omega$ is nondegenerate on the fibres, the $\omega$-orthogonal complements

$$
\mathcal{H}_{x}:=\left(\operatorname{ker} d_{x} p\right)^{\omega}
$$

to the tangent spaces of the fibres of $p$ define a distribution of horizontal subspaces $\mathcal{H}$ such that

$$
T M=\mathcal{H} \oplus \operatorname{ker} d p .
$$

Horizontal lifts of a path $\gamma:[0,1] \rightarrow B$ with given initial points in $p^{-1}(\gamma(0))$ give rise to the parallel transport

$$
P_{\gamma}: p^{-1}(\gamma(0)) \rightarrow p^{-1}(\gamma(1))
$$

along $\gamma$. Closedness of $\omega$ inplies that $P_{\gamma}$ is symplectic, i.e.

$$
P_{\gamma}^{*} \omega_{\gamma(1)}=\omega_{\gamma(0)}
$$

\footnotetext{
${ }^{2}$ This terminology differs slightly from the one in 15 .
} 
where $\omega_{b}$ denotes the symplectic form $\left.\omega\right|_{p^{-1}(b)}$.

Holonomy. The parallel transport $P_{\gamma}: p^{-1}(\gamma(0)) \rightarrow p^{-1}(\gamma(0))$ along a closed curve $\gamma:[0,1] \rightarrow B$ is called the holonomy of $\omega$ along the loop $\gamma$. If $P_{\gamma}=$ id for each loop $\gamma$ we say that $\omega$ has trivial holonomy. In this case parallel transport along any (not necessarily closed) curve depends only on the end points, so we can use parallel transport to define local trivializations of $p: M \rightarrow B$.

Remark 2.1. There is a natural notion of curvature of a symplectic connection, see [15. This is a 2-form on the base with values in the functions on the fibres which measures the nonintegrability of the horizontal distribution. For simply connected base (which is the case of interest to us) the curvature and the holonomy carry the same information, so in this paper we will phrase everything in terms of holonomy.

From foliations to fibrations. More generally, we can consider a closed 2 -form $\omega$ on $M$ whose restriction to the leaves of a smooth foliation $\mathcal{F}$ of $M$ is nondegenerate. If all leaves of $\mathcal{F}$ are compact, then the space of leaves is a smooth manifold $B$ and the canonical projection $M \rightarrow B$ is a fibration, so we are back in the situation of a symplectic connection as above. Since in our case all leaves will be 2-spheres, we can switch freely between the terminologies of foliations and fibrations.

\subsection{Fibered Lagrangian tori in $S^{2} \times S^{2}$}

Suppose now that $(M, \omega)$ is a symplectic 4-manifold and $p: M \rightarrow B$ is a symplectic fibration over a surface $B$ (i.e., the fibres are symplectic surfaces).

Definition 2.2. We say that an embedded 2-torus $L \subset M$ is fibered by $p$ if (see Figure 2)

(i) $\gamma:=p(L)$ is an immersed loop with transverse self-intersections which are at most double points;

(ii) $p^{-1}(\gamma(t)) \cap L$ is diffeomorphic to a circle if $\gamma(t)$ is not a double point, and to two disjoint circles if $\gamma(t)$ is a double point;

(iii) in each of the circles in $p^{-1}(\gamma(t)) \cap L$ we can fill in an embedded disk $D \subset p^{-1}(\gamma(t))$ in the fibre such that the two disks at a double point are disjoint and all the disks form a solid torus $T \cong S^{1} \times D^{2}$ with $L$ as its boundary.

Suppose now that $L$ is in addition Lagrangian. The following two results are the basis for most of the sequel. The first one states that a fibered Lagrangian torus $L$ is generated by parallel transport along $\gamma$ of the circle in the fibre over a non-double point, see Figure 3 .

Proposition 2.3. Let $L \subset M$ be an embedded Lagrangian torus which is fibered by the symplectic fibration $p: M \rightarrow B$. Then $L$ is invariant under parallel transport along $\gamma=p(L)$ with respect to the symplectic connection $\omega$. 


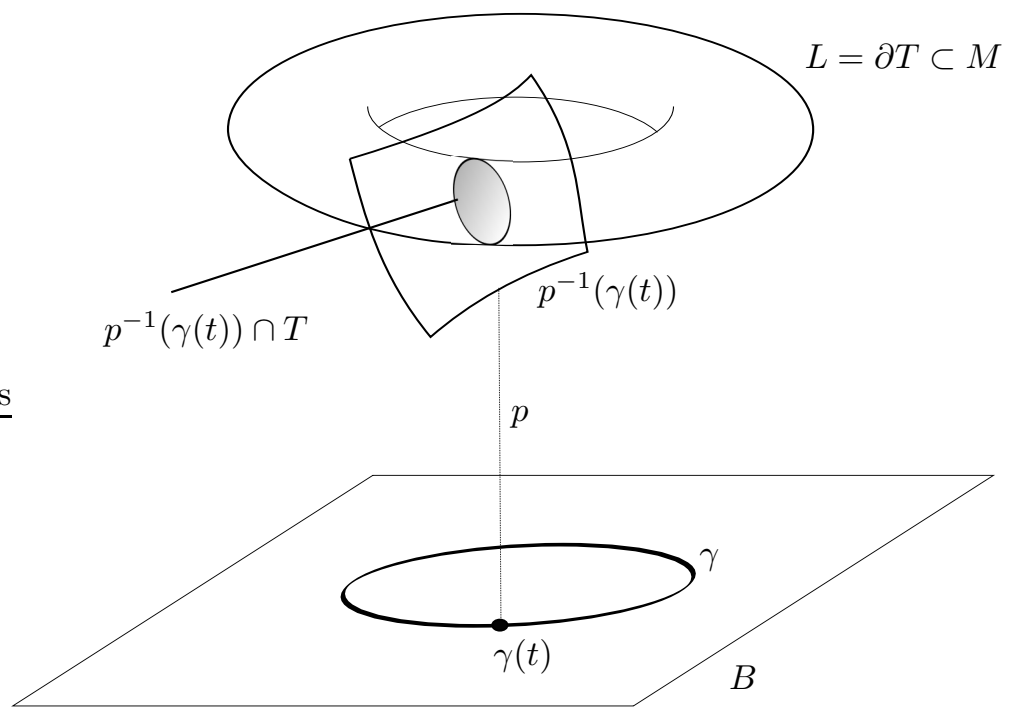

Figure 2: A fibered Lagrangian torus

Proof. At a point $x \in L$ we have the $\omega$-orthogonal splitting $T_{x} M=\mathcal{H}_{x} \oplus V_{x}$, where $V_{x}:=\operatorname{ker} d_{x} p$ denotes the tangent space to the fibre. Since $L$ is fibered by $p$, the subspace $T_{x} L+V_{x} \subset T_{x} M$ generated by $T_{x} L$ and $V_{x}$ is 3 -dimensional. The condition that $L$ is Lagrangian implies $\left(T_{x} L\right)^{\omega}=T_{x} L$, thus $T_{x} L \cap \mathcal{H}_{x}=\left(T_{x} L\right)^{\omega} \cap$ $\left(V_{x}\right)^{\omega}=\left(T_{x} L+V_{x}\right)^{\omega}$ is 1-dimensional. This 1-dimensional subspace therefore contains the horizontal lift of $\dot{\gamma}$ through $x$ and the propsition follows.

Remark 2.4. Let $N:=p^{-1}(\gamma)$ be the 3-dimensional submanifold of $M$ formed by the fibres that meet the torus $L$. In the definition of being fibered by $p$ we did not require the torus to be transverse to the fibres of $p$ in $N$. If, however, $L$ is Lagrangian, then Proposition 2.3 shows that we get this property for free.

Monotone tori in $S^{2} \times S^{2}$. From now on we assume that

$$
M=S^{2} \times S^{2}
$$

and the symplectic form $\omega$ is cohomologous to the product form

$$
\omega_{\text {std }}:=\sigma_{\text {std }} \oplus \sigma_{\text {std }}
$$

where $\sigma_{\text {std }}$ is the standard area form on $S^{2}$ normalized by $\int_{S^{2}} \sigma_{\text {std }}=1$. In other words, we require that

$$
\int_{S^{2} \times \mathrm{pt}} \omega=\int_{\mathrm{pt} \times S^{2}} \omega=1 .
$$

Moreover, we assume that the Lagrangian torus $L$ is monotone, i.e., its Maslov 


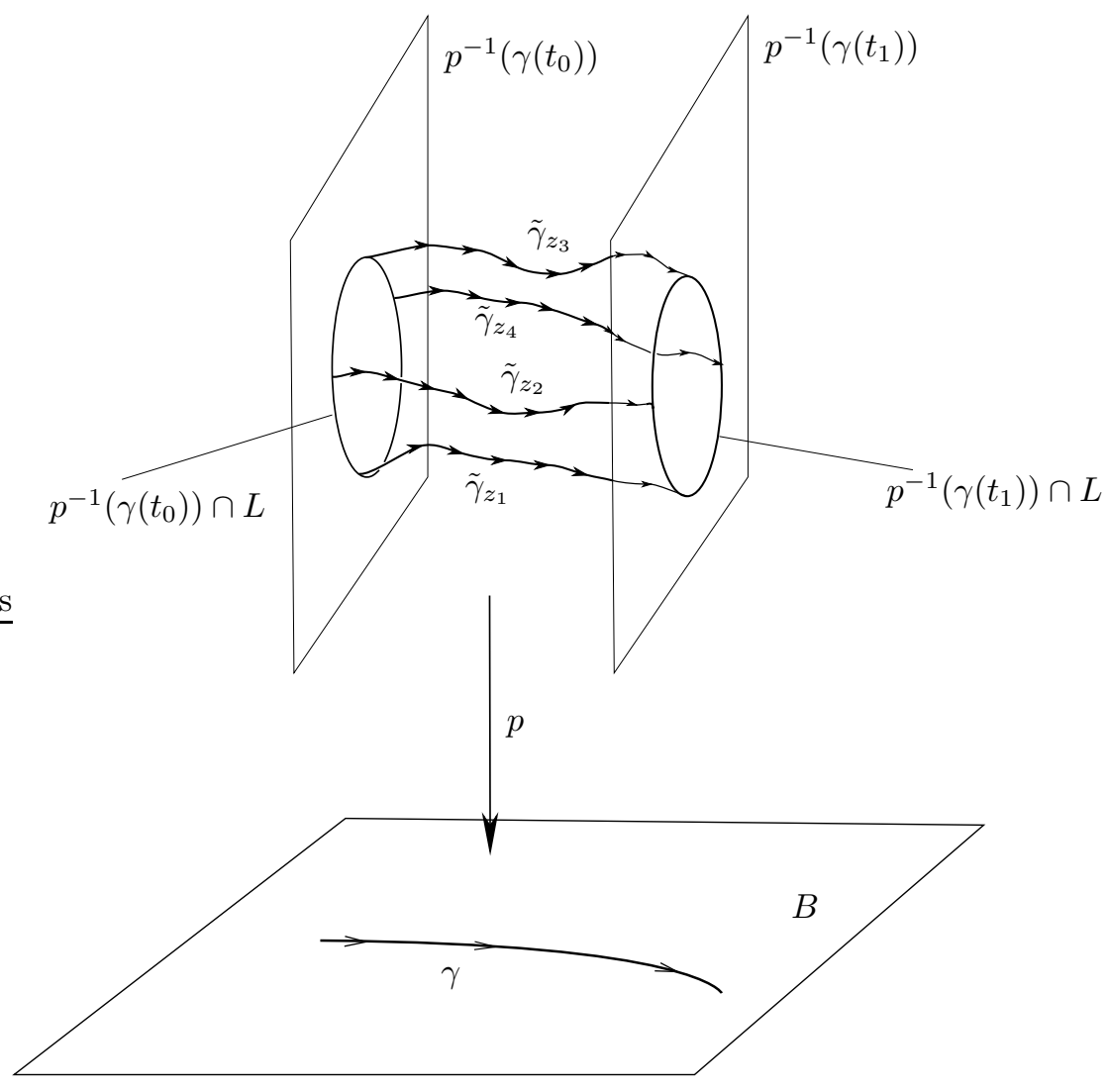

Figure 3: L is generated by parallel transport

class $\mu$ (see [15]) and its symplectic area satisfy

$$
\mu(\sigma)=4 \int_{\sigma} \omega \text { for all } \sigma \in \pi_{2}(M, L) .
$$

Here the monotonicity constant must be equal to 4 because the class $A=\left[S^{2} \times\right.$ pt $] \in \pi_{2}(M)$ has Maslov index $\mu(A)=2\left\langle c_{1}(T M), A\right\rangle=2\left\langle c_{1}\left(T S^{2}\right),\left[S^{2}\right]\right\rangle=4$.

Lemma 2.5. Let $L \subset\left(M=S^{2} \times S^{2}, \omega\right)$ be a monotone Lagrangian torus with $\omega$ cohomologous to $\omega_{\text {std }}$. Let $p: M \rightarrow B$ be a symplectic fibration over the surface $B$ such that $L$ is fibered by $p$. Then the loop $\gamma:=p(L)$ is an embedded curve, i.e., it has no double points.

Proof. Since $L$ is orientable, all its Maslov indices on $\pi_{2}(M, L)$ are even integers. In view of the monotonicity constant 4 , this implies that the symplectic area of each embedded symplectic disk $D \subset M$ with boundary on $L$ must be a positive multiple of $1 / 2$. If $\gamma$ had a double point $b$, then the solid torus $T$ from 
Definition 2.2 would intersect the fibre $p^{-1}(b)$ in two disjoint symplectic disks, which is impossibe because the fibre has symplectic area 1.

Remark 2.6. (a) For a smooth fibration $p: S^{2} \times S^{2} \rightarrow B$ over a surface $B$, both the fibres and the base are diffeomorphic to $S^{2}$. Indeed, denoting a fibre by $F$, the homotopy exact sequence $\pi_{2}(F) \rightarrow \pi_{2}\left(S^{2} \times S^{2}\right) \rightarrow \pi_{2}(B)$ implies $\pi_{2}(F) \cong \pi_{2}(B) \cong \mathbb{Z}$, so $F$ and $B$ must be diffeomorphic to $S^{2}$ or $\mathbb{R} P^{2}$. Since by the product formula for the Euler characteristic $\chi(F) \chi(B)=\chi\left(S^{2} \times S^{2}\right)=4$, both $F$ and $B$ must be diffeomorphic to $S^{2}$.

(b) For a monotone Lagrangian torus $L$ in $M=S^{2} \times S^{2}$, the third condition in Definition 2.2 is actually a consequence of the first two. To see this, note first that in the proof of Lemma 2.5 we can rule out the double point $b$ without reference to the solid torus $T$ : By the Jordan curve theorem, the two circles in $L \cap p^{-1}(b)$ would bound two disjoint symplectic disks in the fibre $p^{-1}(b) \cong S^{2}$, each of area a positive multiple of $1 / 2$, which again gives the desired contradiction. Now an orientation of $L$ and a parametrization of the curve $\gamma \subset B$ induce via horizontal lifts of $\dot{\gamma}$ orientations of the circles $L_{t}:=L \cap p^{-1}(\gamma(t))$, and we define $T$ as the union of the disks $D_{t} \subset p^{-1}(\gamma(t))$ whose oriented boundary is $L_{t}$.

\subsection{Relative symplectic fibrations of $S^{2} \times S^{2}$}

We continue with the manifold $M=S^{2} \times S^{2}$ and the generators

$$
A=\left[S^{2} \times \mathrm{pt}\right], B=\left[\mathrm{pt} \times S^{2}\right] \in H_{2}(M) .
$$

Now we define the main object of study for this paper.

Definition 2.7. A relative symplectic fibration on $M=S^{2} \times S^{2}$ is a quintuple $\mathfrak{S}=\left(\mathcal{F}, \omega, L, \Sigma, \Sigma^{\prime}\right)$ where

- $\mathcal{F}$ is a smooth foliation of $M$ by 2 -spheres in homology class $B$;

- $\omega$ is a symplectic form on $M$ making the leaves of $\mathcal{F}$ symplectic with $\omega(A)=\omega(B)=1$;

- $\Sigma, \Sigma^{\prime}$ are disjoint symplectic submanifolds in class $A$ which are transverse to all the leaves of $\mathcal{F}$, so in particular the projection $p: M \rightarrow \Sigma$ sending each leaf to its unique intersection point with $\Sigma$ defines a symplectic fibration;

- $L \subset M$ is an embedded monotone Lagrangian torus fibered by $p$;

- $\Sigma^{\prime}$ is disjoint from the solid torus $T$ with $\partial T=L$ in Definition 2.2

- $\Sigma$ intersects each fibre $p^{-1}(\gamma(t))$ in the interior of the disk $T \cap p^{-1}(\gamma(t))$. 
Note that for a monotone Lagrangian torus $L$ fibered by $p: M \rightarrow B$ there always exist disjoint smooth sections $\Sigma, \Sigma^{\prime}$ of $p$ with $\Sigma^{\prime}$ disjoint from the solid torus $T$ and $\Sigma \cap p^{-1}(\gamma)$ contained in the interior of $T$. The crucial condition in Definition 2.7 is that these sections can be chosen to be symplectic.

Definition 2.8. (a) A homotopy of relative symplectic fibrations is a smooth 1-parametric family

$$
\mathfrak{S}_{t}=\left(\mathcal{F}_{t}, \omega_{t}, L_{t}, \Sigma_{t}, \Sigma_{t}^{\prime}\right), \quad t \in[0,1]
$$

of relative symplectic fibrations.

(b) The group Diff $\operatorname{Did}_{(}(M)$ of diffeomorphisms $\phi: M \rightarrow M$ inducing the identity on the second homology group $H_{2}(M)$ (and hence on all homology groups) acts on relative symplectic fibrations by push-forward

$$
\phi(\mathfrak{S}):=\left(\phi(\mathcal{F}), \phi_{*} \omega, \phi(L), \phi(\Sigma), \phi\left(\Sigma^{\prime}\right)\right) .
$$

Two relative symplectic fibrations $\mathfrak{S}$ and $\widetilde{\mathfrak{S}}$ are called diffeomorphic if $\widetilde{\mathfrak{S}}=\phi(\mathfrak{S})$ for a diffeomorphism $\phi$ of $M$ (which then necessarily belongs to $\operatorname{Diff}_{\mathrm{id}}(M)$ ).

(c) Two relative symplectic fibrations $\mathfrak{S}$ and $\widetilde{\mathfrak{S}}$ on $M$ are called deformation equivalent if there exists a diffeomorphism $\phi \in \operatorname{Diff}_{\mathrm{id}}(M)$ such that $\phi(\mathfrak{S})$ is homotopic to $\widetilde{\mathfrak{S}}$.

Remark 2.9. (a) Note that a diffeomorphism $\phi \in \operatorname{Diff}_{\text {id }}(M)$ intertwines the symplectic connections of $\mathfrak{S}$ and $\phi(\mathfrak{S})$ and their parallel transports. For example, $\mathfrak{S}$ has trivial holonomy iff $\phi(\mathfrak{S})$ does.

(b) It is easy to see that deformation equivalence is an equivalence relation. Moreover, $\mathfrak{S}$ is deformation equivalent to $\widetilde{\mathfrak{S}}$ iff there exists a homotopy $\mathfrak{S}_{t}$ such that $\mathfrak{S}_{0}=\mathfrak{S}$ and $\mathfrak{S}_{1}$ is diffeomorphic to $\widetilde{\mathfrak{S}}$.

(c) Note that in the above definition nothing is said about the isotopy class of the diffeomorphism $\phi$. In fact, it is an open problem whether every $\phi \in$ $\operatorname{Diff}_{\mathrm{id}}(M)$ is isotopic to the identity, so we do not know whether diffeomorphic relative symplectic fibrations are homotopic in general. However, by a theorem of Gromov (see Theorem 4.12 below), two diffeomorphic relative symplectic fibrations with the same symplectic form $\omega_{\text {std }}$ are homotopic. This result will be crucial at the end of the proof of our main theorem.

\subsection{The standard relative symplectic fibration}

The standard relative symplectic fibration $\mathfrak{S}_{\mathrm{std}}=\left(\mathcal{F}_{\mathrm{std}}, \omega_{\mathrm{std}}, L_{\mathrm{std}}, S_{0}, S_{\infty}\right)$ of $S^{2} \times S^{2}$ consists of the following data:

- $\mathcal{F}_{\text {std }}$ is the foliation by the fibres $\{z\} \times S^{2}$ of the projection $p_{1}: S^{2} \times S^{2} \rightarrow$ $S^{2}$ onto the first factor;

- $\omega_{\text {std }}=\sigma_{\text {std }} \oplus \sigma_{\text {std }}$ is the standard symplectic form; 
- $S_{0}=S^{2} \times\{S\}$ and $S_{\infty}=S^{2} \times\{N\}$, where $N, S \in S^{2}$ are the north and south poles;

- $L_{\mathrm{std}}=E \times E$ is the Clifford torus, i.e. the product of the equators in the base and fibre;

- $T_{\text {std }}=E \times D_{\text {lh }}$, where $D_{\mathrm{lh}} \subset S^{2}$ denotes the lower hemisphere, is the solid torus bounded by $L_{\text {std }}$.

The main goal of this paper will be to deform a given relative symplectic fibration to the standard one (see Theorem 4.11 below). For later use, let us record the relative homology and homotopy groups of the Clifford torus.

Lemma 2.10. For the Clifford torus $L_{\mathrm{std}} \subset S^{2} \times S^{2}$ the second relative homotopy/homology group

$$
\pi_{2}\left(S^{2} \times S^{2}, L_{\text {std }}\right) \cong H_{2}\left(S^{2} \times S^{2}, L_{\text {std }}\right) \cong H_{2}\left(S^{2} \times S^{2}\right) \oplus H_{1}\left(T^{2}\right)
$$

is free abelian generated by

$$
\left[S^{2} \times \mathrm{pt}\right], \quad\left[\mathrm{pt} \times S^{2}\right], \quad\left[D_{\mathrm{lh}} \times \mathrm{pt}\right],\left[\mathrm{pt} \times D_{\mathrm{lh}}\right] .
$$

Proof. The long exact sequences of the pair $\left(M=S^{2} \times S^{2}, L=L_{\text {std }}\right)$ and the Hurewicz maps yield the commuting diagram

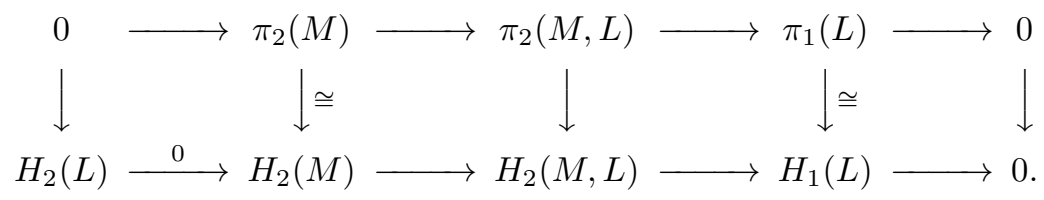

Here the first horizontal map in the lower row is zero because $L$ bounds the solid torus $T_{\mathrm{std}}=E \times D_{\mathrm{lh}}$ in $S^{2} \times S^{2}$, where $E \subset S^{2}$ denotes the equator. Now the middle vertical map is an isomorphism by the five lemma, and the generators of $H_{2}(M, L)$ are obtained from the generators $\left[S^{2} \times \mathrm{pt}\right],\left[\mathrm{pt} \times S^{2}\right]$ of $H_{2}(M)$ and $[E \times \mathrm{pt}],[\mathrm{pt} \times E]$ of $H_{1}(L)$.

\section{Standardisations}

In this section we show how to deform a relative symplectic fibration to make it split (in a sense defined below) near the symplectic sections $\Sigma, \Sigma^{\prime}$ and near one fibre $F$. In particular, the standardised fibration will have trivial holonomy in these regions. This provides a convenient setup for the discussion in Section 4

\subsection{Pullback by diffeomorphisms}

In this subsection, we show how to put a relative symplectic fibration $\mathfrak{S}$ into a nicer form via pullback by diffeomorphisms. Note that this is not really changing 
$\mathfrak{S}$ but just looking at it from a different angle. We will see that using pullbacks we can either standardise all data except the symplectic form $\omega$, or all data except the foliation $\mathcal{F}$. So the nontriviality of a relative symplectic fibration only arises from the interplay of $\omega$ and $\mathcal{F}$, as measured by the holonomy of the corresponding symplectic connection.

In order to establish a clean picture of what can be achieved by pullbacks, we will prove some results in stronger versions than what we actually need in the sequel.

Fixing the fibration. We begin with a useful characterisation of diffeomorphisms that are trivial on homology. Recall the generators $A=\left[S^{2} \times \mathrm{pt}\right]$ and $B=\left[\mathrm{pt} \times S^{2}\right]$ of $H_{2}\left(S^{2} \times S^{2}\right)$.

Lemma 3.1. A diffeomorphism $\phi$ of $S^{2} \times S^{2}$ is trivial on homology if and only if it is orientation preserving and satisfies $\phi_{*}(B)=B$.

Proof. The "only if" is clear, so let us prove the "if". Let us write $\phi_{*}(A)=$ $m A+n B$ for integers $m, n$. Since $\phi$ is orientation preserving, it preserves the intersection form on $H_{2}\left(S^{2} \times S^{2}\right)$ and we obtain

$$
\begin{aligned}
& 1=\phi_{*}(A) \cdot \phi_{*}(B)=(m A+n B) \cdot B=m, \\
& 0=\phi_{*}(A) \cdot \phi_{*}(A)=(A+n B) \cdot(A+n B)=2 n .
\end{aligned}
$$

This shows that $\phi_{*} A=A$, so $\phi_{*}$ is the identity on $H_{2}\left(S^{2} \times S^{2}\right)$.

Our first normalisation result is

Proposition 3.2 (Fixing the fibration). Let $\mathfrak{S}=\left(\mathcal{F}, \omega, L, \Sigma, \Sigma^{\prime}\right)$ be a relative symplectic fibration of $M=S^{2} \times S^{2}$. Then there exists a diffeomorphism $\phi \in$ $\operatorname{Diff}_{\text {id }}(M)$ such that $\phi^{-1}(\mathfrak{S})=\left(\mathcal{F}_{\text {std }}, \widetilde{\omega}, L_{\text {std }}, S_{0}, S_{\infty}\right)$ for some symplectic form $\widetilde{\omega}$.

Proof. Consider the fibration $p: M \rightarrow \Sigma$ defined by $\mathcal{F}$ and pick an orientation preserving diffeomorphism $u: \Sigma \rightarrow S^{2}$. Then $u \circ p: M \rightarrow S^{2}$ is a fibration by 2 -spheres. Since $\pi_{1} D i f f_{+}\left(S^{2}\right)$ classifies $S^{2}$-bundles over $S^{2}$ and $\operatorname{Diff} f_{+}\left(S^{2}\right)$ deformation retracts onto $S O(3)$, there are up to bundle isomorphism precisely two $S^{2}$-bundles over $S^{2}$ : the trivial one $p_{1}: S^{2} \times S^{2} \rightarrow S^{2}$ and a nontrivial one $X \rightarrow S^{2}$. The total space $X$ of the nontrivial bundle is the blow-up of $\mathbb{C} P^{2}$ at one point, which is not diffeomorphic to $S^{2} \times S^{2}$ (e.g. their intersection forms differ). Thus the nontrivial bundle does not occur, and we conclude that there exists a diffeomorphism $\phi: M \rightarrow M$ such the following diagram commutes:

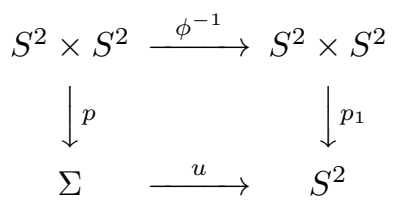


Moreover, the restriction of $\phi$ to each fibre is orientation preserving, which implies the $\phi$ itself is orientation preserving. Since the fibres of $p$ and $p_{1}$ all represent the homology class $B$, it follows that $\phi_{*} B=B$ and thus $\phi \in \operatorname{Diff}_{\text {id }}(M)$ by Lemma 3.1. After replacing $\mathfrak{S}$ by $\phi^{-1}(\mathfrak{S})$, we may hence assume that $\mathcal{F}=$ $\mathcal{F}_{\text {std }}$ and $p=p_{1}$.

The section $\Sigma$ of $p_{1}: S^{2} \times S^{2} \rightarrow S^{2}$ can be uniquely parametrized by $z \mapsto$ $(z, f(z))$ for a smooth map $f: S^{2} \rightarrow S^{2}$. After a preliminary isotopy we may assume that $f\left(z_{0}\right)=S$ equals the south pole $S$ at a base point $z_{0} \in S^{2}$. Then $f$ represents a class in $\pi_{2}\left(S^{2}, S\right)$. Now by Hurewicz's Theorem, $\left.\pi_{2}\left(S^{2}, S\right)\right) \cong$ $H_{2}\left(S^{2}\right)$. Since $[\Sigma]=A=\left[S^{2} \times \mathrm{pt}\right]$, the class of $f$ is trivial in $H_{2}\left(S^{2}\right)$, and thus in $\pi_{2}\left(S^{2}, S\right)$, so that $f$ is nullhomotopic. By smooth approximation, we find a smooth homotopy $f_{t}$ from the constant map $f_{0} \equiv S$ to $f_{1}=f$. Now we use (a fibered version of) the isotopy extension theorem to extend the family of embeddings $S^{2} \times\{S\} \hookrightarrow M,(z, S) \mapsto\left(z, f_{t}(z)\right)$ to a family of fibre preserving diffeomorphisms $\phi_{t}: M \rightarrow M$ with $\phi_{0}=$ id. After replacing $\mathfrak{S}$ by $\phi_{1}^{-1}(\mathfrak{S})$, we may hence assume that $\Sigma=S^{2} \times\{S\}=S_{0}$. Now we repeat the same argument with $\Sigma^{\prime}$ (this time it is even simpler because $S^{2} \backslash\{S\}$ is contractible) to arrange $\Sigma^{\prime}=S^{2} \times\{N\}=S_{\infty}$.

Now the torus $L$ is fibered by $p_{1}: S^{2} \times\left(S^{2} \backslash\{N, S\}\right) \rightarrow S^{2}$. By an isotopy of the base $S^{2}$ we can move the embedded curve $p_{1}(L)$ to the equator $E \subset S^{2}$. Let $e(t), t \in \mathbb{R} / \mathbb{Z}$, be a parametrization of the equator $E$ and consider the loop of embedded closed curves $\Lambda_{t}:=L \cap p_{1}^{-1}(e(t))$ in the fibre $S^{2}$. After a further homotopy we may assume that $\Lambda_{0}=E$. Pick a smooth family of diffeomorphisms $g_{t}: S^{2} \rightarrow S^{2}, t \in[0,1]$, such that $g_{0}=$ id and $g_{t}(E)=\Lambda_{t}$ for all $t$. Moreover, we can arrange that $g_{t}(N)=N$ and $g_{t}(S)=S$ for all $t$. Then $g_{1}$ satisfies $g_{1}(E)=E$ as well as $g_{1}(N)=N$ and $g_{1}(S)=S$. We can alter $g_{t}$ so that $g_{1}$ fixes $E$ pointwise. By a theorem of Smale [17, the group Diff $\left(D^{2}, \partial D^{2}\right)$ of diffeomorphsms of the disk that are the identity near the boundary is contractible. So we can alter $g_{t}$ further (applying this to the upper and lower hemispheres) so that $g_{1}=\mathrm{id}$. This may first destroy the conditions $g_{t}(N)=N$ and $g_{t}(S)=S$, but they can be reinstalled by a further alteration. Now we again use (a fibered version of) the isotopy extension theorem to extend the embedding $E \times S^{2} \hookrightarrow M,(e(t), w) \mapsto\left(e(t), g_{t}(w)\right)$ to a fibre preserving diffeomorphism $\phi: M \rightarrow M$ isotopic to the identity. Then $\phi^{-1}(\mathfrak{S})$ has the desired properties and the proposition is proved.

A similar (in fact, simpler) proof yields the following 1-parametric version of Proposition 3.2 .

Proposition 3.3 (Fixing the fibration - parametric version). Let $\mathfrak{S}_{t}=\left(\mathcal{F}_{t}, \omega_{t}\right.$, $\left.L_{t}, \Sigma_{t}, \Sigma_{t}^{\prime}\right)_{t \in[0,1]}$ be a homotopy of relative symplectic fibrations of $M=S^{2} \times S^{2}$. Then there exists an isotopy of diffeomorphisms $\phi_{t} \in \operatorname{Diff}_{\mathrm{id}}(M)$ with $\phi_{0}=\mathrm{id}$ such that $\phi_{t}^{-1}\left(\mathfrak{S}_{t}\right)=\left(\mathcal{F}_{0}, \widetilde{\omega}_{t}, L_{0}, \Sigma_{0}, \Sigma_{0}^{\prime}\right)$ for some family of symplectic forms $\widetilde{\omega}_{t}$.

Fixing the symplectic form. Our next result is an easy consequence of 
Moser's and Banyaga's theorems. Since it will be used repeatedly in this paper, let us recall the latter [2, Théorème II.2.1] for future reference.

Theorem 3.4 (Banyaga's isotopy extension theorem [2]). Let $(M, \omega)$ be a symplectic manifold and $\psi_{t}: M \rightarrow M$ a smooth isotopy with $\psi_{0}=$ id such that each $\psi_{t}$ is symplectic on a neighbourhood of a compact subset $X \subset M$. Suppose that $\int_{\sigma} \psi_{t}^{*} \omega$ is constant in $t$ for each $\sigma \in H_{2}(M, X)$. Then there exists a symplectic isotopy $\phi_{t}$ with $\phi_{0}=\mathrm{id}$ and $\left.\phi_{t}\right|_{X}=\left.\psi_{t}\right|_{X}$.

Proposition 3.5 (Fixing the symplectic form - parametric version). Let $\mathfrak{S}_{t}=$ $\left(\mathcal{F}_{t}, \omega_{t}, L_{t}, \Sigma_{t}, \Sigma_{t}^{\prime}\right)_{t \in[0,1]}$ be a homotopy of relative symplectic fibrations of $M=$ $S^{2} \times S^{2}$. Then there exists an isotopy of diffeomorphisms $\phi_{t} \in \operatorname{Diff}_{\mathrm{id}}(M)$ with $\phi_{0}=$ id such that $\phi_{t}^{-1}\left(\mathfrak{S}_{t}\right)=\left(\widetilde{\mathcal{F}}_{t}, \omega_{0}, L_{0}, \Sigma_{0}, \Sigma_{0}^{\prime}\right)$ for some family of foliations $\widetilde{\mathcal{F}}_{t}$.

Proof. First, Moser's theorem provides an isotopy of diffeomorphisms $\phi_{t}: M \rightarrow$ $M$ with $\phi_{0}=$ id such that $\phi_{t}^{*} \omega_{t}=\omega_{0}$. After replacing $\mathfrak{S}_{t}$ by $\phi^{-1}\left(\mathfrak{S}_{t}\right)$, we may hence assume that $\omega_{t}=\omega_{0}$ for all $t$.

Next, consider the isotopy of submanifolds $X_{t}:=L_{t} \amalg \Sigma_{t} \amalg \Sigma_{t}^{\prime}$ of $\left(M, \omega_{0}\right)$. Let us write (using the smooth isotopy extension theorem) $X_{t}=\psi_{t}\left(X_{0}\right)$ for diffeomorphisms $\psi_{t}: M \rightarrow M$ with $\psi_{0}=$ id. Since $L_{t}$ is Lagrangian and $\Sigma_{t} \amalg \Sigma_{t}^{\prime}$ is symplectic, the Lagrangian and symplectic neighbourhood theorems provide a modification of $\psi_{t}$ which is symplectic on a neighbourhood of $X_{0}$.

We claim that the symplectic area $\int_{\sigma_{t}} \omega_{0}$ is constant in $t$ for each $\sigma \in H_{2}\left(M, X_{0}\right)$, where we denote $\sigma_{t}:=\left(\psi_{t}\right)_{*} \sigma \in H_{2}\left(M, X_{t}\right)$. To see this, note that the map $H_{2}\left(M, L_{0}\right) \rightarrow H_{2}\left(M, X_{0}\right)$ is surjective because $H_{1}\left(\Sigma_{0} \amalg \Sigma_{0}^{\prime}\right)=0$. So it suffices to prove the claim for classes $\sigma \in H_{2}\left(M, L_{0}\right) \cong \pi_{2}\left(M, L_{0}\right)$. Now recall that the Lagrangian tori $L_{t}$ are monotone with respect to $\omega_{0}$. Since the Maslov class

$\mu\left(\sigma_{t}\right)$ of $L_{t}$ is constant in $t$, so is the symplectic area $\int_{\sigma_{t}} \omega_{0}$ by monotonicity and the claim is proved.

In view of the claim, $\left(X_{0}, \psi_{t}\right)$ satisfies the hypotheses of Banyaga's Theorem 3.4 It follows that the smooth isotopy $\psi_{t}$ can be altered to a symplectic isotopy $\phi_{t}$ with $\phi_{0}=$ id and $\phi_{t}\left(X_{0}\right)=X_{t}$. This is the desired isotopy in the proposition.

A non-parametric version of Proposition 3.5 is much more subtle and will be discussed in Section 4.8

\subsection{Standardisation near a fibre}

Let us pick the point $z_{0}:=(1,0,0)$ on the equator $E$ in the base, so that $F:=p_{1}^{-1}\left(z_{0}\right)$ is a fibre of $\mathcal{F}_{\text {std }}$ intersecting $L_{\text {std }}$ in the equator $E$. The following proposition shows that we can deform every relative symplectic fibration to make the triple $(\mathcal{F}, \omega, L)$ standard near $F$. 
Proposition 3.6 (Standardisation near a fibre). Every relative symplectic fibration $\mathfrak{S}=\left(\mathcal{F}_{\text {std }}, \omega, L_{\text {std }}, \Sigma, \Sigma^{\prime}\right)$ is homotopic to a fibration of the form $\widetilde{\mathfrak{S}}=$ $\left(\mathcal{F}_{\text {std }}, \widetilde{\omega}, L_{\text {std }}, \widetilde{\Sigma}, \widetilde{\Sigma}^{\prime}\right)$ such that $\widetilde{\omega}=\omega_{\text {std }}$ on a neighbourhood of the fibre $F$.

The proof is given in [13, Lemma 3.2.3]. For convenience, we recall the argument. It is based on two easy lemmas.

Lemma 3.7. Let $E$ denote the equator and $D_{\mathrm{lh}}$ the lower hemisphere in $S^{2} \subset$ $\mathbb{R}^{3}$. Let $\sigma$ be a symplectic form on $S^{2}$ cohomologous to $\sigma_{\mathrm{std}}$ such that $\int_{D_{\mathrm{lh}}} \sigma=\frac{1}{2}$. Then there exists an isotopy of diffeomorphisms $h_{t}: S^{2} \rightarrow S^{2}$ with $h_{0}=\mathrm{id}$ such that $h_{t}(E)=E$ for all $t$ and $h_{1}^{*} \sigma=\sigma_{\text {std }}$.

Proof. We apply Moser's theorem to $\sigma_{t}:=(1-t) \sigma_{\text {std }}+t \sigma$ to find a diffeotopy $f_{t}$ : $S^{2} \rightarrow S^{2}$ with $f_{t}^{*} \sigma_{t}=\sigma_{\text {std }}$. Since $\int_{D_{\mathrm{lh}}} \sigma_{t}=1 / 2$ for all $t$, the $\sigma_{\text {std-Lagrangians }}$ $f_{t}^{-1}(E)$ all bound disks of $\sigma_{\text {std }}$-area $1 / 2$. Hence Banyaga's Theorem 3.4 yields

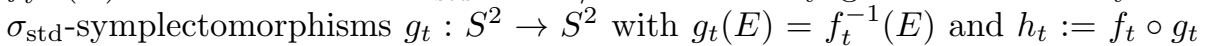
is the desired diffeotopy.

Lemma 3.8. Let $\omega$ be a symplectic form on $M=S^{2} \times S^{2}$ compatible with the standard fibration $p_{1}: M \rightarrow S^{2}$. Let $\delta \subset S^{2}$ be an embedded closed arc passing through $z_{0}$. Then every symplectomorphism $h:\left(F, \sigma_{\text {std }}\right) \rightarrow\left(F,\left.\omega\right|_{F}\right)$ extends to a diffeomorphism $\psi$ between neighbourhoods of $p_{1}^{-1}(\delta)$ preserving the fibres over $\delta$ and such that $\psi^{*} \omega=\omega_{\text {std }}$.

Proof. Parallel transport in $N:=p_{1}^{-1}(\delta)$ with respect to $\omega_{\text {std }}$ from $p_{1}^{-1}(z)$ to $F$ and then with respect to $\omega$ from $F$ to $p_{1}^{-1}(z)$ yields a fibre preserving diffeomorphism $\phi: N \rightarrow N$ extending $h$ with $\phi^{*}\left(\left.\omega\right|_{N}\right)=\left.\left(\omega_{\text {std }}\right)\right|_{N}$. By the coisotropic neighbourhood theorem, $\phi$ extends to the desired diffeomorphism $\psi$.

Proof of Proposition [3.6. By Lemma 3.7 there exists a diffeomorphism $h: F \rightarrow$ $F$ with $h(E)=E$ and $h^{*}\left(\left.\omega\right|_{F}\right)=\sigma_{\text {std }}$. Let $\delta \subset E$ be an arc in the equator in the base passing though $z_{0}$. By Lemma 3.8, the diffeomorphism $h$ extends to a diffeomorphism $\psi$ between neighbourhoods of $p_{1}^{-1}(\delta)$ preserving the fibres over $\delta$ and such that $\psi^{*} \omega=\omega_{\text {std. }}$. Thus the pullback fibration $\psi^{*} \mathcal{F}_{\text {std }}$ and the pullback torus $\psi^{-1}\left(L_{\text {std }}\right)$ coincide over $\delta$ with $\mathcal{F}_{\text {std }}$ and $L_{\text {std }}$, respectively (the latter holds because $\psi^{-1}\left(L_{\text {std }}\right)$ is obtained by parallel transport of $\left\{z_{0}\right\} \times E$ along $\delta$ ). Since $h: F \rightarrow F$ is isotopic to the identity through diffeomorphisms preserving $E$, we can restrict $\psi$ to a smaller neighbourhood $V=p_{1}^{-1}\left(V^{\prime}\right)$ of $F$ and extend it from there to a diffeomorphism $\phi: M \rightarrow M$ preserving $L_{\text {std }}$ (and which equals the identity outside a larger neighbourhood of $F$ ). Then the pullback $\phi^{-1}(\mathfrak{S})$ satisfies $\left.\left(\phi^{*} \omega\right)\right|_{V}=\omega_{\text {std }}$ and $\phi^{-1}(\mathcal{F})=\mathcal{F}_{\text {std }}$ on $p_{1}^{-1}(\delta) \cap V$.

So far we have just put $\mathfrak{S}$ into a more convenient form by a diffeomorphism, but now we will change it. Note that the fibres of $\mathcal{F}_{\text {std }}$ near $p_{1}^{-1}(\delta) \cap V$ are $C^{1}$-close to those of $\phi^{-1}(\mathcal{F})$ and therefore symplectic for $\phi^{*} \omega$. Hence we can deform $\phi^{-1}(\mathcal{F})$ to a foliation $\overline{\mathcal{F}}$, keeping it $\omega_{\text {std }}$-symplectic and fixed on $p_{1}^{-1}(\delta) \cap V$ and outside $V$, such that $\overline{\mathcal{F}}=\mathcal{F}_{\text {std }}$ on a neighbourhood $U \subset V$ of $F$. Thus 
we have constructed a homotopy from $\mathfrak{S}$ to $\overline{\mathfrak{S}}=\left(\overline{\mathcal{F}}, \phi^{*} \omega, L_{\text {std }}, \bar{\Sigma}, \bar{\Sigma}^{\prime}\right)$ such that $\left(\overline{\mathcal{F}}, \phi^{*} \omega\right)=\left(\mathcal{F}_{\text {std }}, \omega_{\text {std }}\right)$ on $U$. Finally, we apply Proposition 3.3 to this homotopy outside the set $U$ to replace it by one with fixed foliation $\mathcal{F}_{\text {std }}$ (as well as $L_{\text {std }}$ which was fixed already). The end point of this homotopy is the desired relative symplectic foliation $\widetilde{\mathfrak{S}}$.

Remark 3.9. Even if in Proposition 3.6 the sections $\Sigma, \Sigma^{\prime}$ in $\mathfrak{S}$ are the standard sections $S_{0}, S_{\infty}$, this will not be true for the sections in $\widetilde{\mathfrak{S}}$ unless the original sections were horizontal near $F$. This will be remedied in the following subsection.

\subsection{Standardisation near the sections}

Consider a relative symplectic fibration of the form $\mathfrak{S}=\left(\mathcal{F}_{\text {std }}, \omega, L_{\text {std }}, S_{0}, S_{\infty}\right)$ with the the projections $p_{1}, p_{2}: S^{2} \times S^{2}$ onto the two factors.

Definition 3.10. We say that $\omega$ is split on a neighbourhood

$$
W=\left(U_{F} \times S^{2}\right) \cup\left(S^{2} \times U_{0}\right) \cup\left(S^{2} \times U_{\infty}\right)
$$

of $F \cup S_{0} \cup S_{\infty}$ if there exist symplectic forms $\sigma_{0}, \sigma_{\infty}$ on $S^{2}$ such that

$$
\begin{aligned}
& \omega=p_{1}^{*} \sigma_{0}+p_{2}^{*} \sigma_{\text {std }} \quad \text { on the set } \quad W_{0}=\left(U_{F} \times S^{2}\right) \cup\left(S^{2} \times U_{0}\right), \quad \text { and } \\
& \omega=p_{1}^{*} \sigma_{\infty}+p_{2}^{*} \sigma_{\text {std }} \quad \text { on the set } \quad W_{\infty}=\left(U_{F} \times S^{2}\right) \cup\left(S^{2} \times U_{\infty}\right) .
\end{aligned}
$$

Here the forms $\sigma_{0}$ and $\sigma_{\infty}$ may differ, but they agree on $U_{F}$. Note that if $\omega$ is split, then in particular the sections $S_{\infty}$ and $S_{0}$ are horizontal. Moreover, parallel transport of the symplectic connection defined by $\omega$ equals the identity on the region where $\omega$ is split.

The following is the main result of this section.

Proposition 3.11 (Standardisation near a fibre and the sections). Every relative symplectic fibration $\mathfrak{S}=\left(\mathcal{F}_{\text {std }}, \omega, L_{\mathrm{std}}, S_{0}, S_{\infty}\right)$ is homotopic to one of the form $\widetilde{\mathfrak{S}}=\left(\mathcal{F}_{\text {std }}, \widetilde{\omega}, L_{\text {std }}, S_{0}, S_{\infty}\right)$ such that $\widetilde{\omega}$ is split on a neighbourhood $W$ of $F \cup S_{0} \cup S_{\infty}$.

The proof of this proposition will occupy the remainder of this section. Standardisation near a symplectic section is more subtle than near a fibre because the section need not be horizontal, so it takes a large deformation to make it symplectically orthogonal to the fibres.

We first consider the local situation in $\mathbb{R}^{4} \cong \mathbb{C}^{2}$ with the standard symplectic form $\Omega_{0}=d x \wedge d y+d u \wedge d v$ in coordinates $z=x+i y, w=u+i v$. Let $S=\{w=$ $f(z)\}$ be the graph over the $z$-plane of a smooth function $f=g+i h: \mathbb{R}^{2} \rightarrow \mathbb{R}^{2}$ with $f(0)=0$. Orient $S$ by projection onto the $z$-plane. The pullback of $\Omega_{0}$ under the embedding $F(z)=(z, f(z))$ equals

$$
F^{*} \Omega_{0}=d x \wedge d y+d g \wedge d h=(1+\operatorname{det} D f) d x \wedge d y .
$$


Thus $S$ is symplectic (with the given orientation) iff

$$
1+\operatorname{det} D f>0 \text {. }
$$

For a smooth function $\phi:[0, \infty) \rightarrow \mathbb{R}$ consider the new function

$$
\tilde{f}(z):=\phi(|z|) f(z) .
$$

We now derive the condition on $\phi$ such that the graph of $\tilde{f}$ is symplectic. We will see that it suffices to do this for linear maps $f$, so suppose that $f(z)=A z$ for a $2 \times 2$ matrix $A$. We compute for $r:=|z|>0$ :

$$
\begin{aligned}
D \tilde{f}(z) & =\phi(r) D f(z)+\phi^{\prime}(r) f(z)\left(\frac{z}{r}\right)^{t}=\phi(r) A+\frac{\phi^{\prime}(r)}{r} A z z^{t} \\
& =A\left(\phi(r) 11+\frac{\phi^{\prime}(r)}{r} z z^{t}\right) .
\end{aligned}
$$

Since

$$
\begin{aligned}
\operatorname{det}\left(\phi(r) 11+\frac{\phi^{\prime}(r)}{r} z z^{t}\right) & =\operatorname{det}\left(\begin{array}{cc}
\phi+\frac{\phi^{\prime}}{r} x^{2} & \frac{\phi^{\prime}}{r} x y \\
\frac{\phi^{\prime}}{r} x y & \phi+\frac{\phi^{\prime}}{r} y^{2}
\end{array}\right) \\
& =\phi^{2}+\frac{\phi \phi^{\prime}}{r}\left(x^{2}+y^{2}\right)=\phi^{2}+r \phi \phi^{\prime},
\end{aligned}
$$

we have $\operatorname{det} D \tilde{f}=\left(\phi^{2}+r \phi \phi^{\prime}\right) \operatorname{det} A$. This proves

Lemma 3.12. Let $f(z)=A z$ be a linear function $\mathbb{R}^{2} \rightarrow \mathbb{R}^{2}$ with $1+\operatorname{det} A \geq$ $\varepsilon>0$. Let $\phi:[0, \infty) \rightarrow \mathbb{R}$ be a smooth function with $\phi(0)=\phi^{\prime}(0)=0$. Then the graph of $\tilde{f}(z):=\phi(|z|) f(z)$ is symplectic provided that for all $r>0$,

$$
0 \leq \phi(r)^{2}+r \phi(r) \phi^{\prime}(r)<\frac{1}{1-\varepsilon} .
$$

Lemma 3.13. For every $0<\varepsilon<1$ and $\delta>0$ there exists a smooth family of nondecreasing functions $\phi_{s}:[0, \infty) \rightarrow[0,1], s \in[0,1]$, satisfying (2) such that $\phi_{s}(r)=s$ for $r \leq \delta$ and $\phi_{s}(r)=1$ for $r \geq 2 \delta / \sqrt{\varepsilon}$.

Proof. For $r>0$ define $\psi(r)$ by $\psi(r):=r^{2} \phi(r)^{2}$. Then $\psi^{\prime}=2 r\left(\phi^{2}+r \phi \phi^{\prime}\right)$, so (2) is equivalent to

$$
\psi^{\prime}(r)<\frac{2 r}{1-\varepsilon} .
$$

This will be satisfied if $\psi$ solves the differential equation

$$
\psi^{\prime}(r)=\frac{2 r}{1-\varepsilon / 4} .
$$

Then $\psi(r)=r^{2} /(1-\varepsilon / 4)+c$ for some constant $c$ and

$$
\phi^{2}(r)=\frac{1}{1-\varepsilon / 4}+\frac{c}{r^{2}} .
$$


We fix the constant $c$ by $\phi(\delta)=0$ to $c=-\delta^{2} /(1-\varepsilon / 4)$ and obtain

$$
\phi^{2}(r)=\frac{1-\delta^{2} / r^{2}}{1-\varepsilon / 4}
$$

This is an increasing function with $\phi(\delta)=0$ and $\phi(\gamma)=1$ at the point $\gamma=$ $2 \delta / \sqrt{\varepsilon}$. Now observe that if a solution of (2) satisfies $\phi\left(r_{0}\right) \geq 0$ and $\phi^{\prime}\left(r_{0}\right) \geq 0$ for some $r_{0}>0$, then we can decrease the slope to 0 near $r_{0}$ and extend $\phi$ by $\phi(r)=\phi\left(r_{0}\right)$ for $r \geq r_{0} 0$ (or $r \leq r_{0}$ ) to a smooth solution of (2). Applying this procedure at $r_{0}=\delta$ and $r_{0}=\gamma$ yields the desired function $\phi_{0}$ for $s=0$. For $s>0$, we obtain $\phi_{s}$ by smoothing the function $\max \left(s, \phi_{0}\right)$.

Lemma 3.14. Let $\Lambda \subset \mathbb{R}^{2}$ be compact and $\left(S^{\lambda}\right)_{\lambda \in \Lambda}$ be a smooth foliation of a region in $\left(\mathbb{R}^{4}, \Omega_{0}\right)$ by symplectic surfaces $S^{\lambda}$ intersecting the symplectic plane $\{0\} \times \mathbb{R}^{2}$ transversely in $(0, \lambda)$. Then for every neighbourhood $W \subset \mathbb{R}^{4}$ of $\{0\} \times \Lambda$ there exists a neighbourhood $U \subset W$ of $\{0\} \times \Lambda$ and a family of foliations $\left(S_{s}^{\lambda}\right)_{s \in[0,1], \lambda \in \Lambda}$ with the following properties (see Figure 4 ):

(i) $S_{0}^{\lambda}=S^{\lambda}$ and $S_{s}^{\lambda}=S^{\lambda}$ outside $W$;

(ii) $S_{s}^{\lambda}$ is symplectic and intersects $\{0\} \times \mathbb{R}^{2}$ transversely in $(0, \lambda)$;

(iii) $S_{1}^{\lambda}=\mathbb{R}^{2} \times\{\lambda\}$ in $U$.

Moreover, for every $\lambda$ with $S^{\lambda}=\mathbb{R}^{2} \times\{\lambda\}$ in $W$ we have $S_{s}^{\lambda}=S^{\lambda}$ for all $s$.

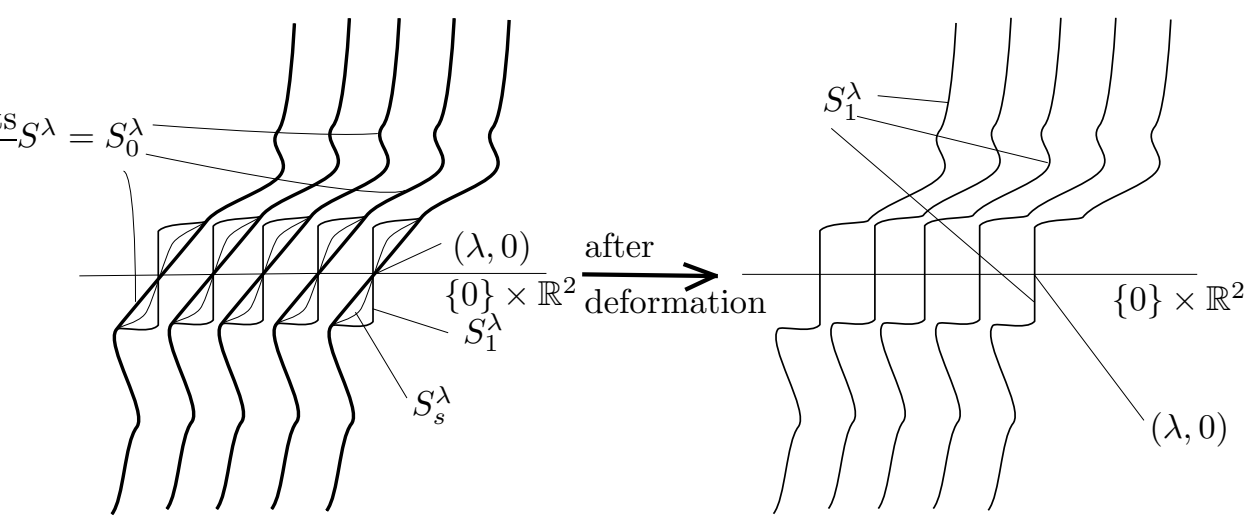

Figure 4: The family of foliations $S_{s}^{\lambda}$

Proof. After shrinking $W$, we may assume that in $W$ each surface can be written as a graph $S^{\lambda}=\left\{w=\lambda+f^{\lambda}(z)\right\}$ over the $z$-plane with $f^{\lambda}(0)=0$. After a $C^{1}$-small perturbation of the surfaces in $W$ (which keeps them symplectic) we may assume that the $f^{\lambda}$ are linear functions $f^{\lambda}(z)=A^{\lambda} z$. Symplecticity implies $\operatorname{det} A^{\lambda}>-1$. Since $\Lambda$ is compact, there exists an $\varepsilon>0$ with $\operatorname{det} A^{\lambda} \geq-1+\varepsilon$ 
in $W$ for all $\lambda$. Moreover, we may assume that the $\varepsilon$-neighbourhood of $\Lambda$ is contained in $W$. Pick $\delta>0$ so small that $2 \delta / \sqrt{\varepsilon}<\varepsilon$. Let $\phi_{s}:[0, \infty) \rightarrow[0,1], s \in$ $[0,1]$, be the functions of Lemma 3.13 and define $f_{s}^{\lambda}(z):=\phi_{1-s}(|z|) f^{\lambda}(z)$. By Lemma 3.12, the graph $S_{s}^{\lambda}$ of $f_{s}^{\lambda}$ satisfies conditions (i)-(iii) of the proposition, where $U$ is the $\delta$-neighbourhood of $\Lambda$. Note that if $S^{\lambda}=\mathbb{R}^{2} \times\{\lambda\}$ for some $\lambda$, then $f^{\lambda}(z) \equiv 0$ and thus $S_{s}^{\lambda}=S^{\lambda}$ for all $s$.

It only remains to verify that the surfaces $\left(S_{s}^{\lambda}\right)_{\lambda \in \Lambda}$ form a foliation for each $s$, or equivalently, that the map $F_{s}: B^{2}(\varepsilon) \times \Lambda \rightarrow \mathbb{R}^{4}$,

$$
F_{s}(z, \lambda):=\left(z, \lambda+f_{s}^{\lambda}(z)\right)=\left(z, \lambda+\phi_{1-s}(|z|) A^{\lambda} z\right),
$$

is an embedding. For injectivity, suppose that $F_{s}(z, \lambda)=F_{s}\left(z^{\prime}, \lambda^{\prime}\right)$. Then $z=z^{\prime}$ and $\lambda-\lambda^{\prime}=-\phi_{1-s}(|z|)\left(A^{\lambda}-A^{\lambda^{\prime}}\right) z$. This implies

$$
\left|\lambda-\lambda^{\prime}\right| \leq\left\|A^{\lambda}-A^{\lambda^{\prime}}\right\||z| \leq \varepsilon\left\|A^{\lambda}-A^{\lambda^{\prime}}\right\| .
$$

Since $A^{\lambda}$ depends smoothly on $\lambda$, there exists a constant $C$ such that $\| A^{\lambda}-$ $A^{\lambda^{\prime}} \| \leq C\left|\lambda-\lambda^{\prime}\right|$. For $\varepsilon<1 / C$ it follows that $\lambda=\lambda^{\prime}$. For the immersion property, consider the differential

$$
D F_{s}(z, \lambda)=\left(\begin{array}{cc}
1 & 0 \\
D_{z} f_{s}^{\lambda} & 11+B_{s}
\end{array}\right), \quad B_{s}=\frac{\partial f_{s}^{\lambda}}{\partial \lambda} .
$$

This is invertible iff the matrix

$$
1+B_{s}=\mathbb{1}+\phi_{1-s}(|z|) \frac{\partial A^{\lambda}}{\partial \lambda} z
$$

is invertible. By smoothness in $\lambda$, there exists a constant $C$ with $\left\|\frac{\partial A^{\lambda}}{\partial \lambda} z\right\| \leq C|z|$. Then for $\varepsilon<1 / C$ we get

$$
\left\|\phi_{1-s}(|z|) \frac{\partial A^{\lambda}}{\partial \lambda} z\right\| \leq C|z| \leq C \varepsilon<1,
$$

which implies invertibility of $11+B_{s}$.

Proof of Proposition 3.11, We deform the given relative symplectic fibration $\mathfrak{S}=\left(\mathcal{F}_{\text {std }}, \omega, L_{\text {std }}, S_{0}, S_{\infty}\right)$ in 4 steps.

Step 1. By Proposition 3.6, $\mathfrak{S}$ is homotopic to $\widetilde{\mathfrak{S}}=\left(\mathcal{F}_{\text {std }}, \widetilde{\omega}, L_{\text {std }}, \widetilde{\Sigma}, \widetilde{\Sigma}^{\prime}\right)$ such that $\widetilde{\omega}=\omega_{\text {std }}$ on a neighbourhood of the fibre $F=\left\{z_{0}\right\} \times S^{2}$. The sections $\widetilde{\Sigma}, \widetilde{\Sigma}^{\prime}$ intersect the fibre in points $\left(z_{0}, q\right)$ and $\left(z_{0}, q^{\prime}\right)$. After pulling back $\widetilde{\mathfrak{S}}$ by a symplectomorphism $(z, w) \mapsto(z, g(w))$, where $g: S^{2} \rightarrow S^{2}$ is a Hamiltonian diffeomorphism preserving the equator and mapping the south pole $S$ to $q$ and the north pole $N$ to $q^{\prime}$, we may assume in addition that $\widetilde{\Sigma} \cap F=\left(z_{0}, S\right)$ and $\widetilde{\Sigma}^{\prime} \cap F=\left(z_{0}, N\right)$.

Step 2. Consider the symplectic section $\widetilde{\Sigma}$. By Lemma 3.14 (with $\Lambda=\{0\}$, $S^{0}=\widetilde{\Sigma}$ and $F=\{0\} \times \mathbb{R}^{2}$ in local coordinates), we can deform $\widetilde{\Sigma}$ such that it 
agrees with $S_{0}=S^{2} \times\{S\}$ near $\widetilde{\Sigma} \cap F$. Since the section $\widetilde{\Sigma}$ is isotopic to $S_{0}$, there exists a diffeomorphism of $S^{2} \times S^{2}$, isotopic to the identity and fixed near $F$, mapping $S_{0}$ to $\widetilde{\Sigma}$. After pulling back everything by this diffeomorphism, we may assume that $\widetilde{\Sigma}=S_{0}$. As in the proof of Proposition 3.6. using the symplectic neighbourhood theorem, by pulling back by an isotopy of $S^{2} \times S^{2}$ fixed near $F$ we can arrange in addition that $\widetilde{\omega}=\omega_{\text {std }}$ near $\widetilde{\Sigma}=S_{0}$ (but the foliation becomes non-standard). The same arguments apply to the other section $\widetilde{\Sigma}^{\prime}$. Altogether, we have shown that $\widetilde{\mathfrak{S}}$ is homotopic to a relative symplectic fibration of the form $\widehat{\mathfrak{S}}=\left(\widehat{\mathcal{F}}, \widehat{\omega}, L_{\text {std }}, S_{0}, S_{\infty}\right)$ with the following properties: $\widehat{\omega}=\omega_{\text {std }}$ and $\widehat{\mathcal{F}}=\mathcal{F}_{\text {std }}$ near the fibre $F=\left\{z_{0}\right\} \times S^{2}$, and $\widehat{\omega}=\omega_{\text {std }}$ near the symplectic sections $S_{0}$ and $S_{\infty}$

Step 3. Next, we adjust the foliation $\widehat{\mathcal{F}}$ near $S_{0} \cup S_{\infty}$. Consider first $S_{0}$. Take a compact subset $\Lambda \subset S^{2} \backslash\left\{z_{0}\right\}$ such that $\widehat{\mathcal{F}}=\mathcal{F}_{\text {std }}$ on a neighbourhood of $\left(S^{2} \backslash\right.$ $\operatorname{int} \Lambda) \times S^{2}$. We identify $\Lambda$ with a subset of $\left(\mathbb{R}^{2}, d x \wedge d y\right)$, and a neighbourhood of $\Lambda \times\{S\}$ in $S^{2} \times S^{2}$ with a neighbourhood $W$ of $\{0\} \times \Lambda$ in $\left(\mathbb{R}^{4}, \Omega_{0}\right)$, by a symplectomorphism of the form $(z, w) \mapsto(f(w), g(z))$. Under this identification, $\widehat{\mathcal{F}}$ corresponds to a symplectic foliation of $W$ transverse to $\{0\} \times \Lambda$ and standard near $\partial \Lambda \times \mathbb{R}^{2}$. By Lemma 3.14, $\widehat{\mathcal{F}}$ can be deformed in $W$, keeping it fixed near $\partial \Lambda \times \mathbb{R}^{2}$, to a symplectic foliation that is standard on a neighbourhood $U$ of $\{0\} \times \Lambda$ in $\mathbb{R}^{4}$. Transfering back to $S^{2} \times S^{2}$ and performing the same construction near $S_{\infty}$, we have thus deformed $\widehat{\mathfrak{S}}$ to a relative symplectic fibration $\overline{\mathfrak{S}}=$ $\left(\overline{\mathcal{F}}, \bar{\omega}, L_{\text {std }}, S_{0}, S_{\infty}\right)$ satisfying $\bar{\omega}=\omega_{\text {std }}$ and $\overline{\mathcal{F}}=\mathcal{F}_{\text {std }}$ near the set $F \cup S_{0} \cup S_{\infty}$. This was the main step. It only remains to deform $\overline{\mathcal{F}}$ back to $\mathcal{F}_{\text {std }}$.

Step 4. By construction, the foliation $\overline{\mathcal{F}}$ is obtained in steps 2 and 3 from $\mathcal{F}_{\text {std }}$ by a homotopy $\mathcal{F}_{t}$ with $\mathcal{F}_{0}=\mathcal{F}$ and $\mathcal{F}_{1}=\overline{\mathcal{F}}$ which is fixed outside $V \backslash V_{F}$, for some neighbourhoods $V$ of $S_{0} \cup S_{\infty}$ and $V_{F}$ of $F$. So we can write $\mathcal{F}_{t}=\phi_{t}\left(\mathcal{F}_{\text {std }}\right)$ for diffeomorphisms with $\phi_{0}=$ id and $\phi_{t}=$ id outside $V \backslash V_{F}$. Since $\overline{\mathcal{F}}$ agrees with $\mathcal{F}_{\text {std }}$ on a smaller neighbourhood $V_{0} \cup V_{\infty}$ of $S_{0} \cup S_{\infty}$, the diffeomorphisms $\phi_{t}$ can be chosen of the form $(z, w) \mapsto\left(z, f_{t}(w)\right)$ on $V_{0}$ and $(z, w) \mapsto\left(z, g_{t}(w)\right)$ on $V_{\infty}$ for diffeomorphisms $f_{t}, g_{t}$ of $S^{2}$. Now the homotopy $\phi_{t}^{-1}(\overline{\mathcal{F}})$ connects $\overline{\mathcal{F}}$ to the symplectic fibration $\phi_{1}^{-1} \overline{\mathfrak{S}}=\left(\mathcal{F}_{\text {std }}, \phi_{1}^{*} \bar{\omega}, L_{\text {std }}, S_{0}, S_{\infty}\right)$, where $\phi_{1}^{*} \bar{\omega}$ is split near $F \cup S_{0} \cup S_{\infty}$. This concludes the proof of Proposition 3.11

Remark 3.15. Replacing Step 4 of the preceding proof by a more careful deformation of the foliation $\overline{\mathcal{F}}$ (not by diffeomorphisms but keeping it symplectic for $\bar{\omega}$ ), we could arrange $\widetilde{\omega}=\omega_{\text {std }}$ near $F \cup S_{0} \cup S_{\infty}$ in Proposition 3.11 As the class of split forms is better suited for the modifications in the next section, we content ourselves with making $\widetilde{\omega}$ split near $F \cup S_{0} \cup S_{\infty}$.

\section{Killing the holonomy}

In this section we will deform a relative symplectic fibration to kill all the holonomy and conclude the proof of the main theorem. A crucial ingredient is the inflation procedure from [14]. 


\subsection{Setup}

Recall that $\mathcal{F}_{\text {std }}$ is the foliation on $S^{2} \times S^{2}$ given by the fibres of the projection $p_{1}$ onto the first factor, $S_{0}=S^{2} \times\{S\}$ and $S_{\infty}=S^{2} \times\{N\}$ are the standard sections, $F=p_{1}^{-1}\left(z_{0}\right)$ is the fibre over the point $z_{0}=(1,0,0)$, and the Clifford torus $L_{\text {std }}=E \times E$ is the product of the equators. In the following, we identify $S_{0}$ with the base $S^{2}$ of the projection $p_{1}$, i.e., we identify $p_{1}$ with the map $(z, w) \mapsto(z, S)$ sending each fibre to its intersection with $S_{0}$.

Our starting point is a relative symplectic fibration $\mathfrak{S}=\left(\mathcal{F}_{\text {std }}, \omega, L_{\text {std }}, S_{0}, S_{\infty}\right)$ as provided by Proposition 3.11 such that $\omega$ is split on a neighbourhood $W=$ $\left(U_{F} \times S^{2}\right) \cup\left(S^{2} \times U_{0}\right) \cup\left(S^{2} \times U_{\infty}\right)$ of $F \cup S_{0} \cup S_{\infty}$. In particular the sections $S_{0}, S_{\infty}$ are horizontal for the symplectic connection. After pulling back $\mathfrak{S}$ by a diffeomorphism of the form $(z, w) \mapsto(\phi(z), w)$ (keeping the same notation), we may replace $U_{F}$ by the ball

$$
B:=\left\{(x, y, z) \in S^{2} \mid x \geq-1 / \sqrt{2}\right\}
$$

so that $\omega$ now satisfies

$$
\begin{aligned}
& \omega=p_{1}^{*} \sigma_{0}+p_{2}^{*} \sigma_{\text {std }} \quad \text { on the set } \quad W_{0}=\left(B \times S^{2}\right) \cup\left(S^{2} \times U_{0}\right), \quad \text { and } \\
& \omega=p_{1}^{*} \sigma_{\infty}+p_{2}^{*} \sigma_{\text {std }} \quad \text { on the set } \quad W_{\infty}=\left(B \times S^{2}\right) \cup\left(S^{2} \times U_{\infty}\right) .
\end{aligned}
$$

Consider the usual spherical coodinates $(\lambda, \mu) \in\left[-\frac{\pi}{2}, \frac{\pi}{2}\right] \times[0,2 \pi]$ on the base $S^{2}$ centered at $z_{0}$. Thus $\lambda$ denotes the latitude and $\mu$ the meridian, and $z_{0}$ lies at $(\lambda, \mu)=(0,0)$; see Figure 5 .

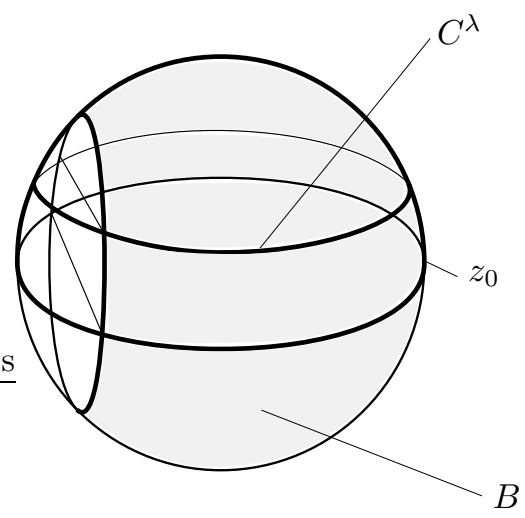

Figure 5: Circles of latitude and the set $B$

Denote by $C^{\lambda}$ the circle of latitude $\lambda$ in the base and by $\phi^{\lambda}$ the symplectic parallel transport around $C^{\lambda}$ parametrised by $\mu \in[0,2 \pi]$. Since the starting and end points of the parametrisation of $C^{\lambda}$ are contained in $B$ for all $\lambda$ and the symplectic form $\omega$ equals $p_{1}^{*} \sigma_{0}+p_{2}^{*} \sigma_{\text {std }}$ over $B$, we can regard $\phi^{\lambda}$ as living in $\operatorname{Symp}\left(S^{2}, \sigma_{\text {std }}\right)$ for all $\lambda$. Moreover, the maps $\phi^{\lambda}$ have the following two properties: 
(i) Since $C^{\lambda} \subset B$ for all $|\lambda| \geq \frac{\pi}{4}$ and the form $\omega$ is split on $B \times S^{2}$, we have $\phi^{\lambda}=$ id for $|\lambda| \geq \frac{\pi}{4}$.

(ii) Since $\omega$ is split on $S^{2} \times\left(U_{0} \cup U_{\infty}\right)$, each $\phi^{\lambda}$ restricts to the identity on $U_{0} \cup U_{\infty}$.

Under stereographic projection $S^{2} \backslash\{N\} \rightarrow \mathbb{C}$ from the north pole $N$, the standard symplectic form on $S^{2}$ corresponds to the form $\sigma_{\text {std }}=\frac{r}{\pi\left(1+r^{2}\right)^{2}} d r \wedge d \theta$ in polar coordinates on $\mathbb{C}$. We pick a closed annulus

$$
A=\{z \in \mathbb{C}|a \leq| z \mid \leq b\} \subset \mathbb{C} \cong S^{2} \backslash\{N\}
$$

with $a>0$ so small and $b>a$ so large that $\partial A \subset U_{0} \cup U_{\infty}$. According to properties (i) and (ii) above, parallel transport along $C^{\lambda}$ then defines maps $\phi^{\lambda} \in \operatorname{Symp}\left(A, \partial A, \sigma_{\text {std }}\right)$ (i.e., symplectomorphisms that equal the identity near $\partial A$, cf. Appendix $\mathrm{A}$ ) that equal the identity for $|\lambda| \geq \frac{\pi}{4}$. In particular, $\left[-\frac{\pi}{2}, \frac{\pi}{2}\right] \ni$ $\lambda \mapsto \phi^{\lambda}$ defines a loop in the identity component $\operatorname{Sym}_{0}\left(A, \partial A, \sigma_{\text {std }}\right)$. Consider the loop of inverses

$$
\psi^{\lambda}=\left(\phi^{\lambda}\right)^{-1} .
$$

Since $L_{\text {std }}=E \times E$ is invariant under parallel transport, the map $\phi^{0}$, and thus $\psi^{0}$, preserves the equator $E$.

\subsection{A special contraction}

According to Proposition A.4 the loop $\psi^{\lambda}$ is contractible in $\operatorname{Symp}_{0}\left(A, \partial A, \sigma_{\text {std }}\right)$. However, in order for the inflation procedure below to work, we need a special contraction $\psi_{s}^{\lambda}$ with the property that $\psi_{s}^{0}(E)=E$ for all $s \in[0,1]$. Here we identify the equator $E$ in $S^{2}$ via stereographic projection with the circle $E=\{|z|=1\} \subset A$.

Proposition 4.1. There exists a smooth contraction $\psi_{s}^{\lambda} \in \operatorname{Symp}_{0}\left(A, \partial A, \sigma_{\mathrm{std}}\right)$ of the loop $\psi^{\lambda}$, with $(s, \lambda) \in[0,1] \times\left[\frac{-\pi}{2}, \frac{\pi}{2}\right]$, such that:

(i) $\psi_{0}^{\lambda}=\mathrm{id}$ and $\psi_{1}^{\lambda}=\psi^{\lambda}$ for all $\lambda$;

(ii) $\psi_{s}^{\lambda}=\mathrm{id}$ for $|\lambda| \geq \frac{\pi}{4}$ and all $s$;

(iii) $\psi_{s}^{\lambda}$ is constant in $s$ near $s=0$ and $s=1$;

(iv) $\psi_{s}^{0}(E)=E$ for all $s$.

Proof. Since the holonomy $\psi^{0}$ along the equator in the base preserves the equator $E$ in the fibre, Lemma A.6 provides a path $\alpha(t) \in \operatorname{Symp}_{0}\left(A, \partial A, \sigma_{\text {std }}\right)$ from the identity to $\psi^{0}$ which preserves $E$ for all $t$. We split the loop $\psi^{\lambda}$ into two paths $\delta_{1}:=\left\{\psi^{\lambda}\right\}_{\lambda \in\left[-\frac{\pi}{2}, 0\right]}$ and $\delta_{2}:=\left\{\psi^{\lambda}\right\}_{\lambda \in\left[0, \frac{\pi}{2}\right]}$. Using these, we define two loops $\gamma_{1}:=\delta_{1} * \bar{\alpha}$ and $\gamma_{2}:=\alpha * \delta_{2}$, where $*$ means concatenation of paths and $\bar{\alpha}$ denotes the path $\alpha$ traversed in the opposite direction; see Figure 6 . By 
Proposition A.4 these loops are contractible in $\operatorname{Symp}_{0}\left(A, \partial A, \sigma_{\text {std }}\right)$, so we can fill them by half-disks $D_{1}, D_{2}$ in $\operatorname{Symp}_{0}\left(A, \partial A, \sigma_{\text {std }}\right)$. Gluing these half-disks along $\alpha$ yields a map $\vartheta: D \rightarrow \operatorname{Symp}_{0}\left(A, \partial A, \sigma_{\text {std }}\right)$ from the unit disk $D \subset \mathbb{C}$ which restricts to the loop $\psi^{\lambda}$ on $\partial D$ (starting and ending at $-i$ ) and to the path $\alpha$ on the imaginary axis. The composition of $\vartheta$ with the map

$$
\eta:[0,1] \times\left[-\frac{\pi}{2}, \frac{\pi}{2}\right] \rightarrow D, \quad(s, \lambda) \mapsto(s-1) i+s e^{i(2 \lambda+\pi / 2)}
$$

(see Figure 7) then has properties (i) and (iv) of the proposition. By smoothing and reparametrisation we finally arrange properties (ii) and (iii) to obtain the desired contraction $\psi_{s}^{\lambda}$.

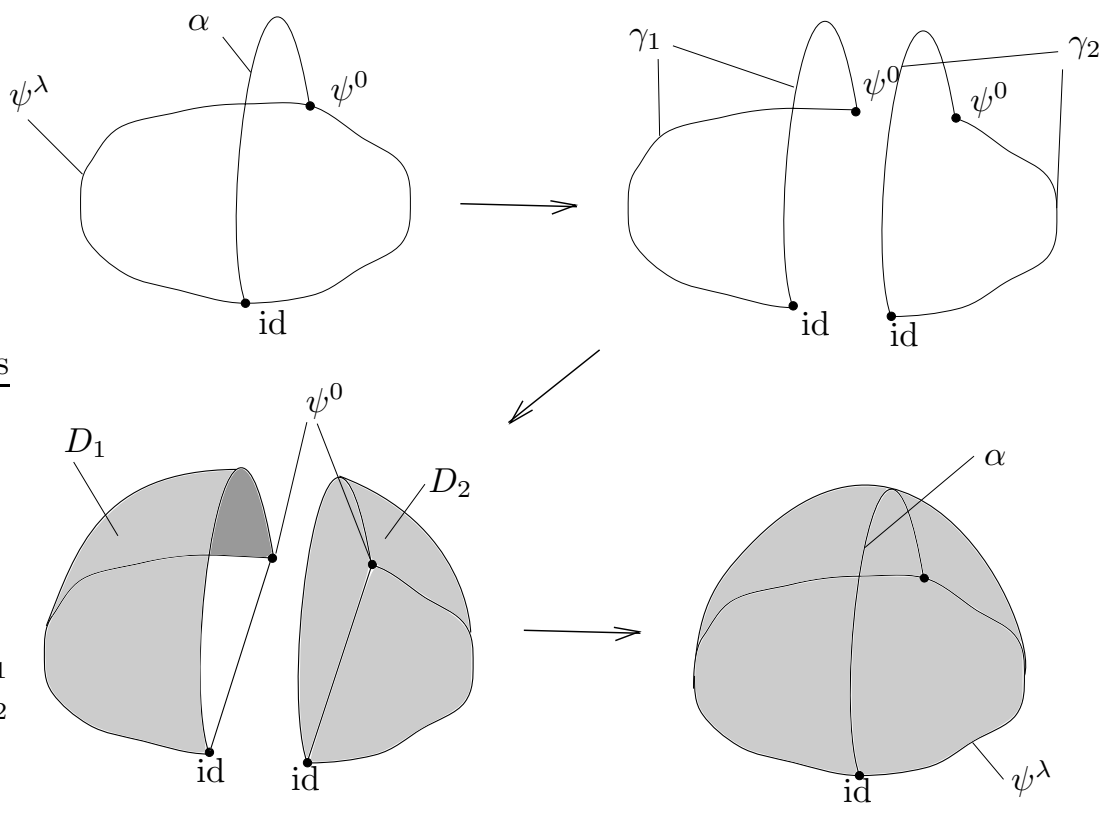

Figure 6: Construction of the special contraction $\psi_{s}^{\lambda}$

\subsection{A special Hamiltonian function}

Next, we construct a family of time-dependent Hamiltonians generating the contraction $\psi_{s}^{\lambda}$ of the previous subsection. We begin with a simple lemma.

Lemma 4.2. Let $(M, \omega=d \lambda)$ be an exact symplectic manifold. Let $\phi_{t}: M \rightarrow$ $M$ be a symplectic isotopy starting at $\phi_{0}=\mathrm{id}$ generated by the time-dependent vector field $X_{t}$, i.e $\frac{d}{d t} \phi_{t}=X_{t} \circ \phi_{t}$. Then $\iota_{X_{t}} \omega=d H_{t}$ for a smooth family of functions $H_{t}: M \rightarrow \mathbb{R}$ if and only if $\phi_{t}^{*} \lambda-\lambda=d F_{t}$ for a smooth family of 

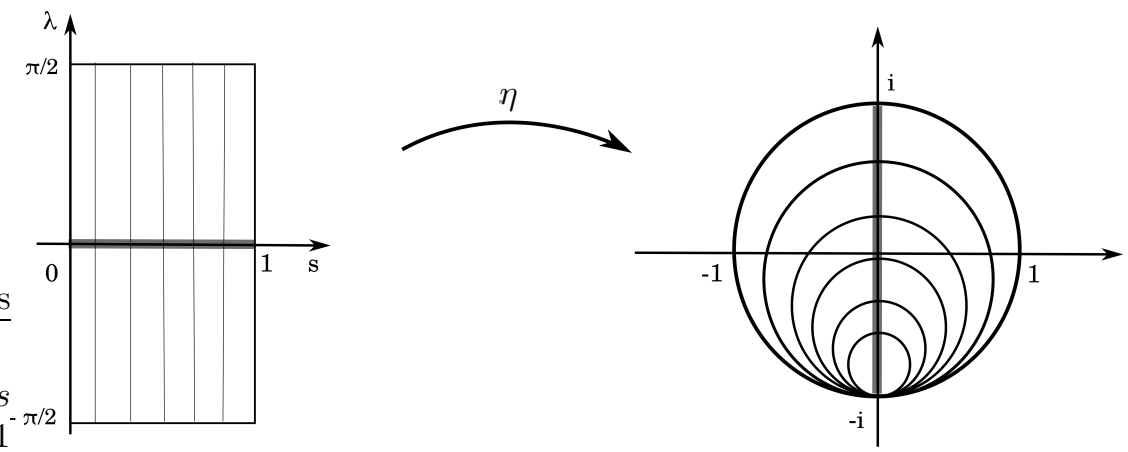

Figure 7: The reparametrisation $\eta$

functions $F_{t}: M \rightarrow \mathbb{R}$. Moreover, $F_{t}$ and $H_{t}$ are related by the equations

$$
F_{t}=\int_{0}^{t}\left(H_{s}+\iota_{X_{s}} \lambda\right) \circ \phi_{s} d s, \quad H_{t}=\dot{F}_{t} \circ \phi_{t}^{-1}-\iota_{X_{t}} \lambda .
$$

Proof. Assume first that $\iota_{X_{t}} \omega=d H_{t}$. Then

$$
\begin{gathered}
\phi_{t}^{*} \lambda-\lambda=\int_{0}^{t} \frac{d}{d s}\left(\phi_{s}^{*} \lambda\right) d s=\int_{0}^{t} \phi_{s}^{*}\left(L_{X_{s}}\right) \lambda d s \\
=\int_{0}^{t} \phi_{s}^{*}\left(\iota_{X_{s}} d \lambda+d \iota_{X_{s}} \lambda\right) d s=d \int_{0}^{t}\left(H_{s}+\iota_{X_{s}} \lambda\right) \circ \phi_{s} d s
\end{gathered}
$$

shows that $\phi_{t}^{*} \lambda-\lambda=d F_{t}$ holds for $F_{t}:=\int_{0}^{t}\left(H_{s}+\iota_{X_{s}} \lambda\right) \circ \phi_{s} d s$. Conversely, if $\phi_{t}^{*} \lambda-\lambda=d F_{t}$, then we differentiate this equation to obtain

$$
d \dot{F}_{t}=\frac{d}{d t}\left(\phi_{t}^{*} \lambda\right)=\phi_{t}^{*}\left(d \iota_{X_{t}} \lambda+\iota_{X_{t}} d \lambda\right),
$$

which shows that $i_{X_{t}} d \lambda=d H_{t}$ holds for $H_{t}:=\dot{F}_{t} \circ \phi_{t}^{-1}-\iota_{X_{s}} \lambda$.

Now let $\psi_{s}^{\lambda} \in \operatorname{Symp}_{0}\left(A, \partial A, \sigma_{\text {std }}\right)$ be the special contraction from Proposition 4.1. Let $\lambda_{\text {std }}=\frac{-1}{2\left(1+r^{2}\right) \pi} d \theta$ be the standard primitive of $\sigma_{\text {std }}$ (any other primitive would also do). Then for each $(s, \lambda)$ the 1 -form $\alpha_{s}^{\lambda}:=\left(\psi_{s}^{\lambda}\right)^{*} \lambda_{\text {std }}-\lambda_{\text {std }}$ on $A$ is closed and vanishes near $\partial A$. So by the relative Poincaré lemma,

$$
\left(\psi_{s}^{\lambda}\right)^{*} \lambda_{\mathrm{std}}-\lambda_{\mathrm{std}}=d F_{s}^{\lambda}
$$

for a unique smooth family of functions $F_{s}^{\lambda}$ that vanish near the lower boundary component $\partial_{-} A=\{a\} \times S^{1}$ of $A$. (We can define $F_{s}^{\lambda}(w):=\int_{\gamma_{w}} \alpha_{s}^{\lambda}$ along any 
path $\gamma_{w}$ from a base point on $\partial_{-} A$ to $w$, which does not depend on the path because every loop can be deformed into $\partial_{-} A$ where $\alpha_{s}^{\lambda}$ vanishes.) Note that $F_{s}^{\lambda}$ will be constant near the upper boundary component $\partial_{+} A=\{b\} \times S^{1}$, where the constant may depend on $s$ and $\lambda$.

By Lemma 4.2 the family $F_{s}^{\lambda}$ is related to a smooth family of Hamiltonians $\widetilde{H}_{s}^{\lambda}$ generating the isotopy $\psi_{s}^{\lambda}$ (for fixed $\lambda$ ) by the formula

$$
\widetilde{H}_{s}^{\lambda}=\frac{\partial F_{s}^{\lambda}}{\partial s} \circ\left(\psi_{s}^{\lambda}\right)^{-1}-\iota_{X_{s}^{\lambda}} \lambda_{\text {std }},
$$

where $\frac{d}{d t} \psi_{t}^{\lambda}=X_{t}^{\lambda} \circ \psi_{t}^{\lambda}$. By construction, $\widetilde{H}_{s}^{\lambda}$ vanishes near the lower boundary component $\partial_{-} A$ of $A$ and it is constant near the upper boundary component $\partial_{+} A$ (where the constant may vary with $s$ and $\lambda$ ). Further, since $\psi_{s}^{\lambda}$ is constant near its ends in both $s$ and $\lambda$, we have $\widetilde{H}_{s}^{\lambda}=0$ for $|\lambda| \geq \frac{\pi}{4}$ and for $s<2 \epsilon$, $s>1-2 \epsilon$ with some $\epsilon>0$.

Note that, since $\psi_{s}^{0}$ preserves the equator, the Hamiltonian vector field $X_{s}^{0}$ is tangent to $E$ for all $s$. So the restriction $\left.\widetilde{H}_{s}^{0}\right|_{E}$ is constant for all $s$ and defines a function $\widetilde{H}_{E}(s)$. For reasons that will become clear in the next subsection, we wish to modify $\widetilde{H}$ to make this function vanish. For this, we pick be a smooth cutoff function $\rho: \mathbb{R} \rightarrow[0,1]$ with $\rho(0)=1$ and support in $\left[-\frac{\pi}{4}, \frac{\pi}{4}\right]$ and define

$$
H_{s}^{\lambda}:=\widetilde{H}_{s}^{\lambda}-\rho(\lambda) \widetilde{H}_{E}(s) .
$$

Since $H_{s}^{\lambda}$ differs from $\widetilde{H}_{s}^{\lambda}$ only by a function of $s$ and $\lambda$, it still has the same Hamiltonian vector field and thus still generates the family $\psi_{s}^{\lambda}$. By construction, $H_{s}^{\lambda}$ depends only on $s$ and $\lambda$ near the boundary $\partial A$ (with possibly different functions at the two boundary components), $H_{s}^{\lambda}=0$ for $|\lambda| \geq \frac{\pi}{4}$ and for $s<2 \epsilon$, $s>1-2 \epsilon$, and

$$
\left.H_{s}^{0}\right|_{E}=0 \text { for all } s .
$$

We define in spherical coordinates on the base the squares

$$
\begin{aligned}
& Q:=\left\{(\mu, \lambda) \in S^{2} \backslash\{N, S\}|2 \epsilon \leq \mu \leq 1-2 \epsilon,| \lambda \mid \leq \frac{\pi}{4}\right\}, \\
& \widetilde{Q}:=\left\{(\mu, \lambda) \in S^{2} \backslash\{N, S\}|\epsilon \leq \mu \leq 1-\epsilon,| \lambda \mid \leq \frac{\pi}{3}\right\} .
\end{aligned}
$$

Note that $Q \subset \operatorname{int} \widetilde{Q}$ and $\widetilde{Q} \subset \operatorname{int} B$, where $B$ is the region defined at the beginning of this section over which $\omega$ is split. The family $H_{s}^{\lambda}$ constructed above gives rise to a smooth function

$$
H: \widetilde{Q} \times A \rightarrow \mathbb{R}, \quad(\lambda, \mu, w) \mapsto H_{\mu}^{\lambda}(w) .
$$

Let us write the fibre sphere as

$$
S^{2}=\operatorname{Cap}_{N} \cup A \cup \operatorname{Cap}_{S},
$$

where $\mathrm{Cap}_{N}$ and $\mathrm{Cap}_{S}$ denote the northern and southern polar caps, respectively. Then we can extend $H$ first to $\widetilde{Q} \times S^{2}$ by the corresponding functions of 
$(\lambda, \mu)$ on the southern and northern polar caps, and then to all of $S^{2} \times S^{2}$ by zero outside $\widetilde{Q} \times S^{2}$. We still denote the resulting function by $H: S^{2} \times S^{2} \rightarrow \mathbb{R}$. By construction, $H$ has support in $Q \times S^{2}$, it depends only on $(\lambda, \mu)$ outside $Q \times A$, and $\left.H(0, \mu)\right|_{E} \equiv 0$ for all $\mu$, where we denote $H(\lambda, \mu):=\left.H\right|_{p_{1}^{-1}(\lambda, \mu)}$.

\subsection{A special symplectic connection}

Recall that we consider a relative symplectic fibration $\left(\mathcal{F}_{\text {std }}, \omega, L_{\text {std }}, S_{0}, S_{\infty}\right)$ such that the symplectic form $\omega$ is split on the set $\left(B \times S^{2}\right) \cup\left(S^{2} \times\left(U_{0} \cup U_{\infty}\right)\right)$. Our current goal is to change the symplectic form $\omega$, in its relative cohomology class in $H^{2}\left(S^{2} \times S^{2}, L_{\text {std }} ; \mathbb{R}\right)$, to a form $\omega^{\prime}$ which has trivial holonomy around the circles of latitude. To explain the idea, consider a circle of latitude $C^{\lambda}$ (cf. Figure 8). As the symplectic form is split over $B$, its parallel transport equals the identity along the part of $C^{\lambda}$ lying within $B$, so the holonomy $\phi^{\lambda}$ is realised by travelling along the part of $C^{\lambda}$ outside $B$.

$$
\phi^{\lambda} \text { is realised here } \quad Q \cap C^{\lambda} ; \psi^{\lambda} \text { shall be realised here }
$$

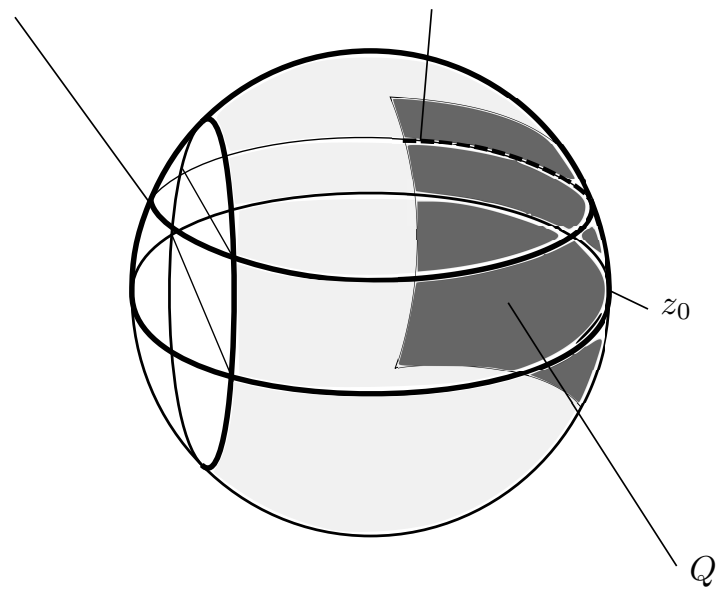

Figure 8: The path $Q \cap C^{\lambda}$ and $B \cap C^{\lambda}$

The idea is now to modify $\omega$ to $\omega^{\prime}$ such that the symplectic connection of $\omega^{\prime}$ agrees with that of $\omega$ outside $Q \times S^{2}$ and realises the inverse holonomy $\psi^{\lambda}$ along $C^{\lambda} \cap Q$ for all $\lambda$.

For the following computations, let us rename the coordinates $(\lambda, \mu)$ to

$$
x:=\mu \in[0,1], \quad y:=\lambda \in\left[-\frac{\pi}{3}, \frac{\pi}{3}\right] .
$$

Recall that the function $H: S^{2} \times S^{2} \rightarrow \mathbb{R}$ constructed in the previous subsection has support in $Q \times S^{2}$, where $Q=[2 \epsilon, 1-2 \epsilon] \times\left[-\frac{\pi}{4}, \frac{\pi}{4}\right]$ in the new coordinates $(x, y)$. Consider the closed 2-form

$$
\Omega_{H}=\omega+d H \wedge d x
$$


on $Q \times A$, extended by $\omega$ to a form on all of $S^{2} \times S^{2}$. Since $\Omega_{H}$ is vertically nondegenerate, the $\Omega_{H}$-orthogonal complements to the tangent spaces of the fibres of $p_{1}$ induce a symplectic connection on $S^{2} \times S^{2}$.

Lemma 4.3. (a) The holonomy of $\Omega_{H}$ along each circle of latitude $C^{\lambda}$ is trivial.

(b) The closed form $\Omega_{H}$ vanishes on $L_{\text {std }}$ and is relatively cohomologous to $\omega$.

Proof. (a) Recall that $H$ depends only on $x$ and $y$ outside the set $Q \times A$, so $\Omega_{H}$ and $\omega$ differ there by the pullback $d H \wedge d x=p_{1}^{*} \alpha$ of a 2-form $\alpha$ from the base. Since adding the pullback of a 2 -form from the base does not change the symplectic connection (because $\iota_{v}\left(p_{1}^{*} \alpha\right)=0$ for every vertical vector $v$ ), the induced connections of $\Omega_{H}$ and $\omega$ agree outside the set $Q \times A$. Within $Q \times A$ the form $\omega=p_{1}^{*} \sigma_{0}+p_{2}^{*} \sigma_{\text {std }}$ is split, so that its induced connection is flat. The horizontal spaces of the induced connection of $\Omega_{H}$ are spanned by the horizontal lifts of the coordinate vector fields $\partial_{x}, \partial_{y}$. These can be easily seen to be

$$
\widetilde{\partial}_{x}=\partial_{x}+X_{H_{x}^{y}}, \quad \widetilde{\partial}_{y}=\partial_{y},
$$

where $X_{H_{x}^{y}}$ is the Hamiltonian vector field of the Hamiltonian function $H_{x}^{y}(w)=$ $H(x, y, w)$ on the annulus $\left(A, \sigma_{\text {std }}\right)$. To see this, let us write $\tilde{\partial}_{x}=\partial_{x}+v_{x}$ with a vertical vector $v_{x}$. This is horizontal iff

$$
0=\Omega_{H}\left(\widetilde{\partial}_{x}, v\right)=\Omega_{H}\left(\partial_{x}, v\right)+\Omega_{H}\left(v_{x}, v\right)=-d H(v)+\sigma_{\text {std }}\left(v_{x}, v\right)
$$

for all vertical vectors $v$, which just means that $v_{x}$ is the Hamiltonian vector field of $H_{x}^{y}$ with respect to $\sigma_{\mathrm{std}}$. A similar calculation shows that $v_{y}=0$.

It follows that the parallel transport of $\Omega_{H}$ along an interval of latitude $C^{\lambda} \cap Q \cong$ $[2 \epsilon, 1-2 \epsilon] \times\{y\}$ is the time-1 map of the Hamiltonian flow of the time-dependent Hamiltonian $H_{s}^{\lambda}$. By construction of $H_{s}^{\lambda}$, this is the inverse $\psi^{\lambda}$ of the holonomy of $\omega$, and thus of $\Omega_{H}$, along the interval $C^{\lambda} \backslash Q$. Hence the total holonomy of $\Omega_{H}$ along each circle of latitude $C^{\lambda}$ is trivial.

(b) By construction, the horizontal vector field $\widetilde{\partial}_{x}=\partial_{x}+X_{H_{x}^{y}}$ is tangent to $L_{\text {std }}$. Let $v$ be the vertical vector field along $L_{\text {std }}$ given by the positively oriented unit tangent vectors to the equators in the fibres. Since $\Omega_{H}\left(\widetilde{\partial}_{x}, v\right)=0$ by definition of horizontality, this shows that $L_{\text {std }}$ is Lagrangian for $\Omega_{H}$. Finally, let us compute the relative homology class of $\Omega_{H}$ in $H^{2}\left(S^{2} \times S^{2}, L_{\text {std }}\right)$. For this, we evaluate $\Omega_{H}$ on the generators of $H_{2}\left(S^{2} \times S^{2}, L_{\text {std }}\right)$ in Lemma 2.10.

$$
\begin{aligned}
\int_{S^{2} \times \mathrm{pt}} \Omega_{H} & =\int_{\mathrm{pt} \times S^{2}} \Omega_{H}=1, \\
\int_{\mathrm{pt} \times D_{\mathrm{lh}}} \Omega_{H} & =\int_{\mathrm{pt} \times D_{\mathrm{lh}}} \omega=\frac{1}{2}, \\
\int_{D_{\mathrm{lh} \times \mathrm{pt}}} \Omega_{H} & =\frac{1}{2}+\int_{(Q \cap\{y \leq 0\}) \times\{e\}} d H \wedge d x=\frac{1}{2}+\int_{0}^{1} H(x, 0, e) d x=\frac{1}{2} .
\end{aligned}
$$


Here in the last equation $e \in E$ is a base point on the equator in the fibre and we have used the normalisation condition $H(x, 0, e)=\left.H(x, 0)\right|_{E} \equiv 0$ from the previous subsection. Since $\omega$ takes the same values on these classes by monotonicity of $L_{\text {std }}$, this shows that the relative cohomology classes of $\Omega_{H}$ and $\omega$ agree.

Let us analyse when the form $\Omega_{H}$ is symplectic. Since it is closed, this is equivalent to the form $\Omega_{H} \wedge \Omega_{H}$ being a volume form on $S^{2} \times S^{2}$. This is clearly satisfied outside the set $Q \times S^{2}$ because there $H \equiv 0$. On the set $Q \times S^{2}$, the form $\omega$ is split of the form $\omega=p_{1}^{*} \sigma_{0}+p_{2}^{*} \sigma_{\text {std }}$. We work in the chosen coordinates $x, y$ and write the form on the base as

$$
\sigma_{0}=f(x, y) d x \wedge d y
$$

with a positive function $f$. A short computation yields

$$
\Omega_{H} \wedge \Omega_{H}=\left(1-\frac{1}{f} \frac{\partial H}{\partial y}\right) \omega \wedge \omega
$$

So $\Omega_{H}$ will be symplectic iff

$$
1-\frac{1}{f} \frac{\partial H}{\partial y}>0
$$

everywhere. A priori, this need not be true for the given function $H$, but it can be remedied by the inflation procedure in the next subsection.

\subsection{Inflation}

In this subsection we recall the inflation procedure of McDuff and Lalonde [14, suitably adapted to our situation. Let $f_{\sigma}, \bar{f}_{\tau}$ be two smooth nonnegative bump functions on $S^{2}$, where we think of $f_{\sigma}$ as living on the fibre sphere and of $\bar{f}_{\tau}$ as living on the base sphere; see Figure 9, We require that

$$
\operatorname{supp}\left(f_{\sigma}\right) \subset\left(U_{0} \cup U_{\infty}\right) \backslash A=\operatorname{Cap}_{S} \amalg \operatorname{Cap}_{N},
$$

where $U_{0}, U_{\infty}$ are the neighbourhoods of $S, N$ over which $\omega$ is split and $A$ is the annulus from the previous subsection, and that

$$
\int_{\mathrm{Cap}_{S}} f_{\sigma} \sigma_{\mathrm{std}}=\int_{\mathrm{Cap}_{N}} f_{\sigma} \sigma_{\mathrm{std}}=\frac{1}{2}
$$

In particular, $\int_{S^{2}} f_{\sigma} \sigma_{\text {std }}=1$. The function $\bar{f}_{\tau}$ is required to have support in $\widetilde{Q}$ and satisfy

$$
\bar{f}_{\tau}(x, y)=\bar{f}_{\tau}(x,-y)
$$

as well as $\left.\bar{f}_{\tau}\right|_{Q} \equiv 1$. We define

$$
f_{\tau}:=\frac{\bar{f}_{\tau}}{a f} \quad \text { with } \quad a:=\int_{\widetilde{Q}} \frac{\bar{f}_{\tau}}{f} \sigma_{0}=\int_{\widetilde{Q}} \bar{f}_{\tau} d x \wedge d y,
$$


where $\sigma_{0}=f(x, y) d x \wedge d y$ as above. Then $\int_{\widetilde{Q}} f_{\tau} \sigma_{0}=\frac{1}{a} \int_{\widetilde{Q}} \bar{f}_{\tau} d x \wedge d y=1$, which by the symmetry of $\bar{f}_{\tau}$ implies

$$
\int_{\widetilde{Q} \cap\{y \geq 0\}} f_{\tau} \sigma_{0}=\frac{1}{a} \int_{\widetilde{Q} \cap\{y \geq 0\}} \bar{f}_{\tau} d x \wedge d y=\frac{1}{2} .
$$

On the fibre

On the base
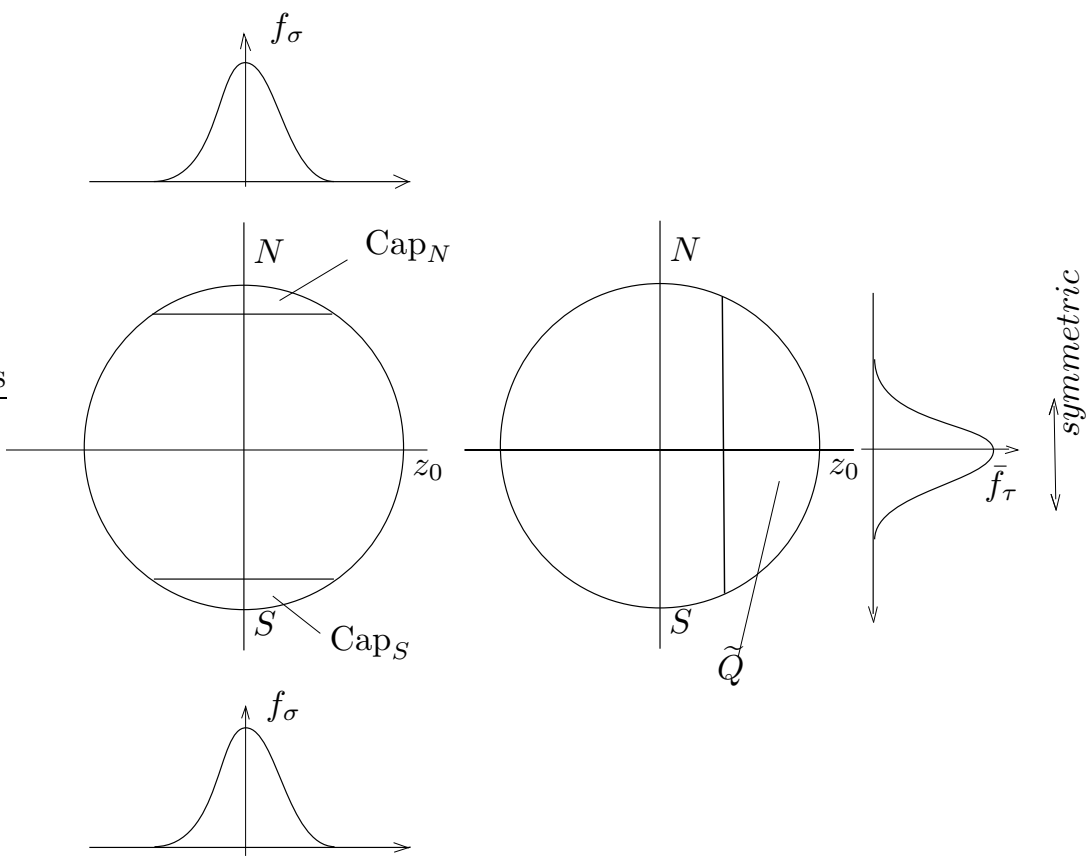

Figure 9: The functions $f_{\sigma}$ and $\bar{f}_{\tau}$

We define the two non-negative 2 -forms

$$
\sigma:=f_{\sigma} \sigma_{\mathrm{std}}, \quad \tau:=f_{\tau} \sigma_{0}
$$

on $S^{2}$ and consider the family of 2 -forms on $S^{2} \times S^{2}$

$$
\omega_{c}:=\frac{1}{c+1}\left(\omega+c p_{1}^{*} \tau+c p_{2}^{*} \sigma\right), \quad c \geq 0
$$

Lemma 4.4. For each $c \geq 0$ the form $\omega_{c}$ has the following properties:

(a) $\omega_{c}$ is symplectic and $L_{\mathrm{std}}$ is Lagrangian for $\omega_{c}$;

(b) $\omega_{c}$ is cohomologous to $\omega_{0}=\omega$ in $H^{2}\left(S^{2} \times S^{2}, L_{\mathrm{std}} ; \mathbb{R}\right)$;

(c) $\omega_{c}$ induces the same symplectic connection as $\omega$. 
Proof. (a) First note that $\omega_{c}$ is closed for all $c \geq 0$ and

$$
\omega_{c}=\frac{1}{c+1} \omega \quad \text { outside } W:=W_{0} \cup W_{\infty},
$$

where $W_{0}, W_{\infty}$ are the sets from (3) on which $\omega$ is split. On the set $W_{0}$,

$$
\omega_{c}=\frac{1}{c+1}\left(\left(1+c p_{1}^{*} f_{\tau}\right) p_{1}^{*} \sigma_{0}+\left(1+c p_{2}^{*} f_{\sigma}\right) p_{2}^{*} \sigma_{\mathrm{std}}\right)
$$

and therefore

$$
\omega_{c} \wedge \omega_{c}=\frac{1}{(c+1)^{2}}\left(1+c p_{1}^{*} f_{\tau}\right)\left(1+c p_{2}^{*} f_{\sigma}\right) \omega \wedge \omega>0
$$

because $c, f_{\tau}, f_{\sigma}$ are nonnegative. For the set $W_{\infty}$, we write $\sigma_{\infty}=g \sigma_{0}$ for a positive function $g$. Then on $W_{\infty}$ we have

$$
\omega_{c}=\frac{1}{c+1}\left(\left(p_{1}^{*} g+c p_{1}^{*} f_{\tau}\right) p_{1}^{*} \sigma_{0}+\left(1+c p_{2}^{*} f_{\sigma}\right) p_{2}^{*} \sigma_{\mathrm{std}}\right),
$$

and again positivity of $g$ and nonnegativity of $c, f_{\tau}, f_{\sigma}$ implies

$$
\omega_{c} \wedge \omega_{c}=\frac{1}{(c+1)^{2}}\left(p_{1}^{*} g+c p_{1}^{*} f_{\tau}\right)\left(1+c p_{2}^{*} f_{\sigma}\right) \omega \wedge \omega>0 .
$$

This proves that $\omega_{c}$ is symplectic. The torus $L_{\text {std }}$ is Lagrangian for $\omega_{c}$ because all pullback forms from the base or the fibre vanish on $L_{\text {std }}$.

(b) To show that $\omega_{c}$ is relatively cohomologous to $\omega$, we evaluate it on the basis of $H_{2}\left(S^{2} \times S^{2}, L_{\text {std }}\right)$ from Lemma 2.10 Using $\int_{S^{2}} \sigma=\int_{S^{2}} \tau=1, \int_{D_{1 \mathrm{~h}}} \sigma=$ $\int_{\operatorname{Cap}_{S}} \sigma=1 / 2$ and $\int_{D_{\mathrm{lh}}} \tau=\int_{\widetilde{Q} \cap\{y \leq 0\}} \tau=1 / 2$, we compute with the point $z_{0} \in E$ on the equator in the base or fibre:

$$
\begin{aligned}
& \int_{\mathrm{pt} \times S^{2}} \omega_{c}=\frac{1}{c+1} \int_{\mathrm{pt} \times S^{2}}\left(\omega+c p_{2}^{*} \sigma\right)=\frac{1}{c+1}(1+c)=1, \\
& \int_{S^{2} \times \mathrm{pt}} \omega_{c}=\frac{1}{c+1} \int_{S^{2} \times \mathrm{pt}}\left(\omega+c p_{1}^{*} \tau\right)=\frac{1}{c+1}(1+c)=1, \\
& \int_{\mathrm{pt} \times D_{\mathrm{lh}}} \omega_{c}=\frac{1}{c+1} \int_{\left\{z_{0}\right\} \times D_{\mathrm{lh}}}\left(\omega+c p_{2}^{*} \sigma\right)=\frac{1}{c+1}\left(\frac{1}{2}+\frac{c}{2}\right)=\frac{1}{2}, \\
& \int_{D_{\mathrm{lh}} \times \mathrm{pt}} \omega_{c}=\frac{1}{c+1} \int_{D_{\mathrm{lh}} \times\left\{z_{0}\right\}}\left(\omega+c p_{1}^{*} \tau\right)=\frac{1}{c+1}\left(\frac{1}{2}+\frac{c}{2}\right)=\frac{1}{2} .
\end{aligned}
$$

By monotonicity of $L_{\text {std }}$, the form $\omega$ takes the same values on these classes, so $\left[\omega_{c}\right]=[\omega] \in H^{2}\left(S^{2} \times S^{2}, L_{\text {std }} ; \mathbb{R}\right)$.

(c) On the set $W=W_{0} \cup W_{\infty}$ the forms $\omega_{c}$ and $\omega$ are both split, hence both symplectic connections are flat and the horizontal subspaces are the tangent spaces to the other cartesian factor. Outside $W$ we have $\omega_{c}=\frac{1}{c+1} \omega$ and, since the symplectic complements to the fibres are not affected by scaling of the symplectic form, the symplectic connections of $\omega_{c}$ and $\omega$ agree here as well. 
A new symplectic connection. Now recall that the function $H$ from the previous section is a pullback from the base outside the set $S^{2} \times A$. On the set $S^{2} \times A$, the function $p_{2}^{*} f_{\sigma}$ vanishes and thus $\omega_{c}=\frac{1}{c+1}\left(\left(1+p_{1}^{*} f_{\tau}\right) p_{1}^{*} \sigma_{0}+p_{2}^{*} \sigma_{\text {std }}\right)$. In particular, on this set the restriction of $\omega_{c}$ to the fibres it is just the standard form $\sigma_{\text {std }}$ scaled by $\frac{1}{c+1}$. Now the fibrewise Hamiltonian vector field of the rescaled function $\frac{1}{c+1} H$ with respect to $\frac{1}{c+1} \sigma_{\text {std }}$ equals the fibrewise Hamiltonian vector field $X_{H_{x}^{y}}$ of $H$ with respect to $\sigma_{\text {std }}$. So the horizontal lift of $\partial_{x}$ with respect to the closed 2 -form

$$
\Omega_{H}^{c}:=\omega_{c}+\frac{1}{c+1} d H \wedge d x
$$

agrees with its horizontal lift $\partial_{x}+X_{H_{x}^{y}}$ with respect to $\Omega_{H}$ (see the proof of Lemma 4.3), and since the horizontal lift of $\partial_{y}$ is $\partial_{y}$ in both cases, we see that $\Omega_{H}^{c}$ and $\Omega_{H}$ define the same symplectic connection for all $c \geq 0$. Moreover, the proof of Lemma 4.3(b) shows that $\Omega_{H}^{c}$ vanishes on $L_{\text {std }}$ and is relatively cohomologous to $\omega_{c}$, and thus to $\omega$ by Lemma 4.4 .

Symplecticity. Again, let us analyse when the form $\Omega_{H}^{c}$ is symplectic. Outside $Q \times S^{2}$, the form $\Omega_{H}^{c}$ is just $\omega_{c}$, which is symplectic by Lemma 4.4. On the set $Q \times S^{2} \subset W_{0}$, using equations (5) and (6) we compute

$$
\begin{aligned}
\omega_{c} \wedge \frac{1}{c+1} d H \wedge d x & =\frac{1}{(c+1)^{2}}\left(-\frac{\partial H}{\partial y}\right)\left(1+c p_{2}^{*} f_{\sigma}\right) d x \wedge d y \wedge p_{2}^{*} \sigma_{\text {std }} \\
& =\frac{1}{2 f(c+1)^{2}}\left(-\frac{\partial H}{\partial y}\right)\left(1+c p_{2}^{*} f_{\sigma}\right) \omega \wedge \omega \\
\Omega_{H}^{c} \wedge \Omega_{H}^{c} & =\omega_{c} \wedge \omega_{c}+2 \omega_{c} \wedge \frac{1}{c+1} d H \wedge d x \\
& =\frac{1}{(c+1)^{2}}\left(1+c p_{1}^{*} f_{\tau}-\frac{1}{f} \frac{\partial H}{\partial y}\right)\left(1+c p_{2}^{*} f_{\sigma}\right) \omega \wedge \omega .
\end{aligned}
$$

Now $1+c p_{2}^{*} f_{\sigma} \geq 1$ for all $c$ by nonnegativity of $c$ and $f_{\sigma}$. Moreover, by the choice of $f_{\tau}$ we have $p_{1}^{*} f_{\tau}=\frac{1}{a f}$ on $Q \times S^{2}$. Hence $\Omega_{H}^{c}$ is symplectic iff

$$
1+\frac{1}{f}\left(\frac{c}{a}-\frac{\partial H}{\partial y}\right)>0
$$

on $Q \times S^{2}$. But this is satisfied for

$$
c \geq C:=a \max _{Q \times S^{2}}\left|\frac{\partial H}{\partial y}\right| .
$$

We summarize the preceding discussion in

Lemma 4.5. The closed 2 -form $\Omega_{H}^{c}$ vanishes on $L_{\text {std }}$, is relatively cohomologous to $\omega_{c}$ (and thus $\omega$ ), and has trivial holonomy along each circle of latitude $C^{\lambda}$ for each $c \geq 0$. Moreover, $\Omega_{H}^{c}$ is symplectic for $c \geq C$ given by (8). 


\subsection{Killing the holonomy along circles of latitude}

We denote the 0 -meridian by $m_{0}:=\left\{(\lambda, \mu) \in S^{2} \mid \mu=0\right\}$ in spherical coordinates. Putting the previous subsections together, we can now prove

Proposition 4.6. Let $\mathfrak{S}=\left(\mathcal{F}_{\mathrm{std}}, \omega, L_{\mathrm{std}}, S_{0}, S_{\infty}\right)$ be a relative symplectic fibration such that $\omega$ is split on a neighbourhood of the fibre $F$ and the sections $S_{0}, S_{\infty}$. Then there exists a homotopy of relative symplectic fibrations $\mathfrak{S}_{t}=\left(\mathcal{F}_{\text {std }}, \omega_{t}, L_{\text {std }}, S_{0}, S_{\infty}\right)$ with $\mathfrak{S}_{0}=\mathfrak{S}$ such that the holonomy of $\mathfrak{S}_{1}$ along the circles of latitude $C^{\lambda}$ is the identity for all $\lambda$. Moreover, $\omega_{1}$ is split near the set $\left(m_{0} \times S^{2}\right) \cup S_{0} \cup S_{\infty}$.

Proof. As explained at the beginning of Section 4.1. we may assume that $\omega$ is split on a set $\left(B \times S^{2}\right) \cup\left(\left(S^{2} \times\left(U_{0} \cup U_{\infty}\right)\right)\right.$, where the ball $B \subset S^{2}$ contains the 0 -meridian $m_{0}$. Let $H$ be the Hamiltonian function constructed in Section 4.3 and let $C$ be the constant defined in (8). For $c \in[0, C]$, let $\omega_{c}$ be the form defined in (44). By Lemma 4.4. $\left(\mathcal{F}_{\text {std }}, \omega_{c}, L_{\text {std }}, S_{0}, S_{\infty}\right)$ gives a homotopy of relative symplectic fibrations from $\mathfrak{S}$ to $\left(\mathcal{F}_{\text {std }}, \omega_{C}, L_{\text {std }}, S_{0}, S_{\infty}\right)$. For $t \in[0,1]$, consider the forms

$$
\Omega_{t H}^{C}=\omega_{C}+\frac{t}{C+1} d H \wedge d x=(1-t) \omega_{C}+t \Omega_{H}^{C}
$$

as in (7) (with $H$ replaced by $t H$ and $c$ by $C$ ). By Lemma 4.5 (applied to $t H$ ), $\left(\mathcal{F}_{\text {std }}, \Omega_{t H}^{C}, L_{\text {std }}, S_{0}, S_{\infty}\right)$ gives a homotopy of relative symplectic fibrations from $\left(\mathcal{F}_{\text {std }}, \omega_{C}, L_{\text {std }}, S_{0}, S_{\infty}\right)$ to $\mathfrak{S}_{1}=\left(\mathcal{F}_{\text {std }}, \Omega_{H}^{C}, L_{\text {std }}, S_{0}, S_{\infty}\right)$. By the same lemma, $\mathfrak{S}_{1}$ has trivial holonomy along all circles of latitude. Hence the concatenation of the previous two homotopies gives the desired homotopy $\mathfrak{S}_{t}$. For the last assertion, simply observe that by construction all symplectic forms in this homotopy agree with the original split form $\omega$ near $\left(m_{0} \times S^{2}\right) \cup S_{0} \cup S_{\infty}$.

Remark 4.7. The point of departure for the preceding subsections was the standardisation provided by Proposition 3.11. If rather than making the symplectic form $\omega$ split we had made it equal to $\omega_{\text {std }}$ near $\left(m_{0} \times S^{2}\right) \cup S_{0} \cup S_{\infty}$ (as suggested in Remark 3.15), then the holonomies $\phi^{\lambda}$ would lie in the subgroup $\operatorname{Ham}\left(A, \partial A, \sigma_{\text {std }}\right) \subset \operatorname{Symp}_{0}\left(A, \partial A, \sigma_{\text {std }}\right)$ of symplectomorphisms generated by Hamiltonians with compact support in $A \backslash \partial A$ and the whole construction could be performed in that subgroup (which is also contractible). However, since we change the normalisation of the Hamiltonians $H_{s}^{\lambda}$ anyway to make them vanish on the equator, we would gain nothing from working in this subgroup.

\subsection{Killing all the holonomy}

Now we will further deform the relative symplectic fibration from the previous subsection to one which has trivial holonomy along all closed curves in the base. We begin with a simple lemma. 
Lemma 4.8. Let $\omega, \omega^{\prime}$ be linear symplectic forms on $\mathbb{R}^{4}$ which define the same orientation and agree on a real codimension one hyperplane $H$. Then $\omega_{t}:=$ $(1-t) \omega+t \omega^{\prime}$ is symplectic for all $t \in[0,1]$.

Proof. Take a symplectic basis $e_{1}, f_{1}, e_{2}, f_{2}$ for $\omega$ such that $e_{1}, f_{1}, e_{2}$ is a basis of $H$. Take a vector $f_{2}^{\prime}=a_{1} e_{1}+b_{1} f_{1}+a_{2} e_{2}+b_{2} f_{2}$ such that $e_{1}, f_{1}, e_{2}, f_{2}^{\prime}$ is a symplectic basis for $\omega^{\prime}$. Since $\omega, \omega^{\prime}$ induce the same orientation, we have $b_{2}>0$, and therefore

$$
\omega\left(e_{2}, f_{2}^{\prime}\right)=b_{2}>0, \quad \omega^{\prime}\left(e_{2}, f_{2}\right)=\frac{1}{b_{2}}>0 .
$$

For $\omega_{t}:=(1-t) \omega+t \omega^{\prime}$ we find

$$
\omega_{t} \wedge \omega_{t}=(1-t)^{2} \omega \wedge \omega+2 t(1-t) \omega \wedge \omega^{\prime}+t^{2} \omega^{\prime} \wedge \omega^{\prime},
$$

and therefore

$$
\begin{aligned}
\omega_{t} \wedge \omega_{t}\left(e_{1}, f_{1}, e_{2}, f_{2}^{\prime}\right)= & 2(1-t)^{2} \omega\left(e_{1}, f_{1}\right) \omega\left(e_{2}, f_{2}^{\prime}\right)+2 t^{2} \omega^{\prime}\left(e_{1}, f_{1}\right) \omega^{\prime}\left(e_{2}, f_{2}^{\prime}\right) \\
& +2 t(1-t)\left(\omega\left(e_{1}, f_{1}\right) \omega^{\prime}\left(e_{2}, f_{2}^{\prime}\right)+\omega\left(e_{2}, f_{2}^{\prime}\right) \omega^{\prime}\left(e_{1}, f_{1}\right)\right) \\
> & 0 .
\end{aligned}
$$

Recall the definition of the 0-meridian $m_{0}$ from the previous subsection.

Proposition 4.9. Let $\mathfrak{S}=\left(\mathcal{F}_{\text {std }}, \omega, L_{\mathrm{std}}, S_{0}, S_{\infty}\right)$ be a relative symplectic fibration which is split near the set $S_{0} \cup S_{\infty} \cup\left(m_{0} \times S^{2}\right)$ and has trivial holonomy around all circles of latitude $C^{\lambda}$. Then there exists a homotopy of relative symplectic fibrations $\mathfrak{S}_{t}=\left(\mathcal{F}_{\text {std }}, \omega_{t}, L_{\text {std }}, S_{0}, S_{\infty}\right)$ with $\omega_{0}=\omega$ and $\omega_{1}=\omega_{\text {std }}$.

Proof. The idea of the proof is to use parallel transport along circles of latitude to define a fibre preserving diffeomorphism $\phi$ of $S^{2} \times S^{2}$ which pulls back the symplectic form $\omega$ to a form which agrees with the standard form $\omega_{\text {std }}$ on $C^{\lambda} \times S^{2}$ for all $\lambda$, and then apply Lemma 4.8 .

For each $\lambda, \mu$ let

$$
P_{\mu}^{\lambda}:\{(\lambda, 0)\} \times S^{2} \rightarrow\{(\lambda, \mu)\} \times S^{2}
$$

be the parallel transport of (the symplectic connection on $p_{1}$ defined by) $\omega$ along the circle of latitude $C^{\lambda}$ from $(\lambda, 0)$ to $(\lambda, \mu)$. Since $\omega$ has trivial holonomy along $C^{\lambda}$, this does not depend on the path in $C^{\lambda}$ and is thus well-defined. Note that, due to the fact that $\omega$ is split near $S_{0} \cup S_{\infty}$, the map $P_{\mu}^{\lambda}$ equals the identity near the north and south poles $N, S$ in the fibre.

We define a fibre preserving diffeomorpism $\phi$ of $S^{2} \times S^{2}$ by parallel transport on the left sphere in Figure 10 with respect to the standard form $\omega_{\text {std }}$ first going backwards along the circle of latitude until we hit the meridian $m_{0}$, then upwards along $m_{0}$ until we hit the north pole $N$, then by the identity to the fibre over the north pole of the right sphere, then by parallel transport with 


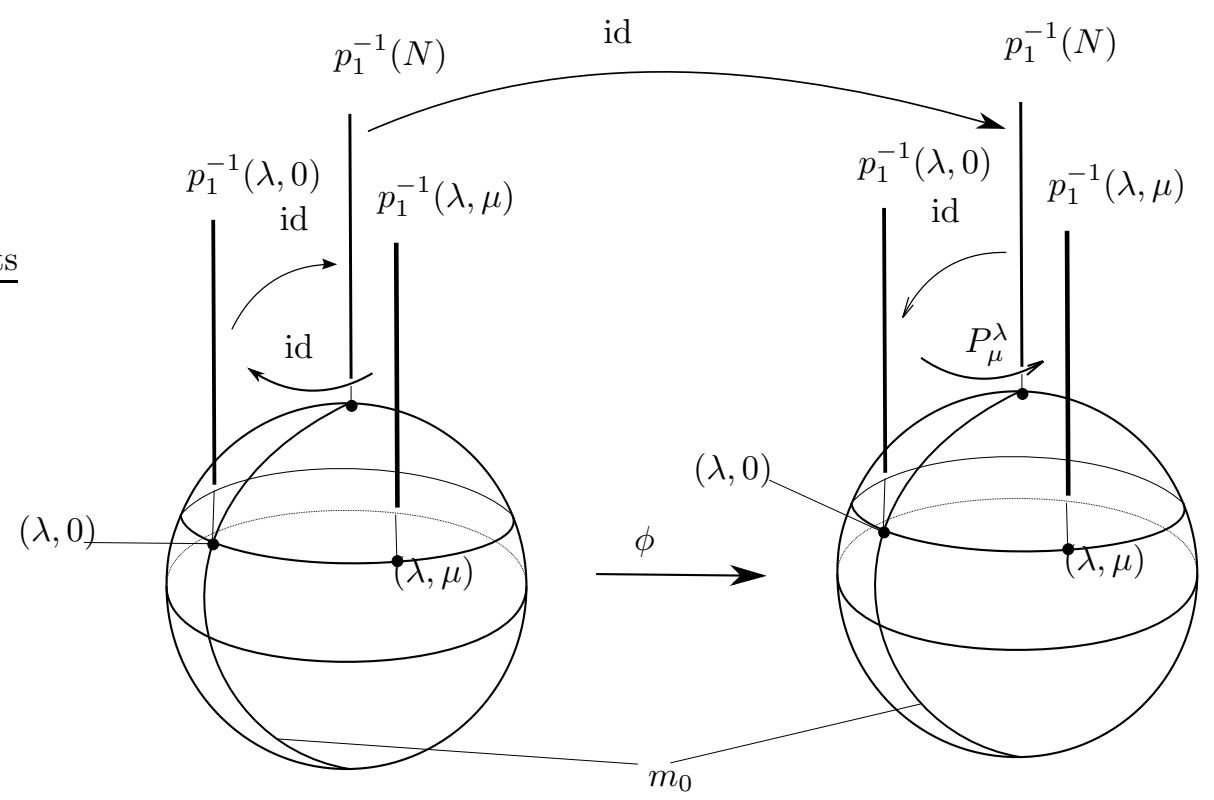

Figure 10: The maps $P_{\mu}^{\lambda}$ and the construction of $\phi$

respect to $\omega$ along $m_{0}$, and finally along the circle of latitude to land in the fibre over the original point $(\lambda, \mu)$. Since parallel transport with respect to the symplectic connection $\omega_{\text {std }}$ is the identity for all paths, as is parallel transport with respect to $\omega$ along paths in $m_{0}$ (since $\omega$ is split over $m_{0}$ ), we will not explicitly include these maps in the notation. Then we can write the preceding definition in formulas as

$$
\phi: S^{2} \times S^{2} \rightarrow S^{2} \times S^{2}, \quad((\lambda, \mu), w) \mapsto\left((\lambda, \mu), P_{\mu}^{\lambda}(w)\right) .
$$

Note that $\phi$ is smooth for $(\lambda, \mu)$ near the north and south poles because there $P_{\mu}^{\lambda}=$ id. For $z \in S^{2}$ let us denote by $\omega_{z}$ the restriction of $\omega$ to the fibre $F_{z}=\{z\} \times S^{2}$. We claim that $\phi$ has the following properties:

(a) $\phi$ restricts to symplectomorphisms $\left(F_{z}, \sigma_{\text {std }}\right) \rightarrow\left(F_{z}, \omega_{z}\right)$ on all fibres;

(b) $\phi$ preserves the Clifford torus $L_{\text {std }}$;

(c) $\phi$ equals the identity near $S_{0} \cup S_{\infty} \cup\left(m_{0} \times S^{2}\right)$;

(d) $\phi$ is isotopic to the identity through fibre preserving diffeomorphisms $\phi_{t}$ that preserve $L_{\text {std }}$ and equal the identity near $S_{0} \cup S_{\infty} \cup\left(m_{0} \times S^{2}\right)$.

For (a), note that $\omega$ restricts to $\sigma_{\text {std }}$ on the fibre $F_{N}$ over the north pole (because $\omega$ is split there), so the identity defines a symplectomorphism $\left(F_{N}, \sigma_{\text {std }}\right) \rightarrow$ $\left(F_{N}, \omega_{N}\right)$. Now (a) follows because parallel transport is symplectic. 
Property (b) follows from the fact that $L_{\text {std }}$ is given by parallel transport of the equator in the fibre around the equator in the base, hence $P_{\mu}^{0}(E)=E$ and thus

$$
\phi(\{(0, \mu)\} \times E)=\{(0, \mu)\} \times P_{\mu}^{0}(E)=\{(0, \mu)\} \times E .
$$

Property (c) holds because $\omega$ is split near $S_{\infty} \cup S_{0} \cup\left(m_{0} \times S^{2}\right)$.

For (d), consider the map

$$
P: R:=\left[-\frac{\pi}{2}, \frac{\pi}{2}\right] \times[0,2 \pi] \rightarrow \operatorname{Diff}(A, \partial A), \quad(\lambda, \mu) \mapsto P_{\mu}^{\lambda} .
$$

It maps the boundary $\partial R$ to id and the interval $\{0\} \times[0,2 \pi]$ to the subspace $\operatorname{Diff}(A, \partial A ; E) \subset \operatorname{Diff}(A, \partial A)$ of diffeomorphisms preserving the equator $E$ (as a set, not pointwise). By Corollary [A.3, the loop $[0,2 \pi] \ni \mu \mapsto P_{\mu}^{0}$ is contractible in $\operatorname{Diff}(A, \partial A ; E)$. Using this and the fact (from Corollary A.2) that $\pi_{2} \operatorname{Diff}(A, \partial A)=0$, we find a contraction of $P$ through maps $P_{t}: R \rightarrow$ $\operatorname{Diff}(A, \partial A)$ sending $\partial R$ to id and $\{0\} \times[0,2 \pi]$ to $\operatorname{Diff}(A, \partial A ; E)$. (The argument is analogous to the proof of Proposition 4.1] with the 1-parametric family $\psi^{\lambda}$ replaced by the 2-parametric family $P_{\mu}^{\lambda}$.) Then $\phi_{t}((\lambda, \mu), w):=$ $\left((\lambda, \mu), P_{t}(\lambda, \mu)(w)\right)$ is the desired isotopy and the claim is proved.

Now we construct the homotopy from $\omega$ to $\omega_{\text {std }}$ in two steps. For the first step, let $\phi_{t}$ be the isotopy in (d) from $\phi_{0}=$ id to $\phi_{1}=\phi$. Then $\phi_{t}^{-1}(\mathfrak{S})=$ $\left(\mathcal{F}_{\text {std }}, \phi_{t}^{*} \omega, L_{\text {std }}, S_{0}, S_{\infty}\right)$ is a homotopy of relative symplectic fibrations from $\mathfrak{S}$ to $\left(\mathcal{F}_{\text {std }}, \phi^{*} \omega, L_{\text {std }}, S_{0}, S_{\infty}\right)$.

For the second step, note that $\phi^{*} \omega$ restricts to $\sigma_{\text {std }}$ on every fibre by property (a). Moreover, since $\phi$ commutes with parallel transport along $C^{\lambda}$ (with respect to $\omega_{\text {std }}$ and $\omega$ ), the horizontal lifts of vectors tangent to circles of latitude with respect to $\omega_{\text {std }}$ and $\phi^{*} \omega$ agree. Accordingly, $\omega_{\text {std }}$ and $\phi^{*} \omega$ agree on the 3-dimensional subspaces $T_{((\lambda, \mu), w)}\left(C^{\lambda} \times S^{2}\right)$ in $T_{((\lambda, \mu), w)}\left(S^{2} \times S^{2}\right)$ for all $((\lambda, \mu), w) \in S^{2} \times S^{2}$. Thus, by Lemma 4.8, the linear interpolations $\omega_{t}:=(1-t) \phi^{*} \omega+t \omega_{\mathrm{std}}$ are symplectic for all $t \in[0,1]$.

We claim that $\left(\mathcal{F}_{\text {std }}, \omega_{t}, L_{\text {std }}, S_{0}, S_{\infty}\right)$ is a relative symplectic fibration for all $t \in[0,1]$. For this, first note that $L_{\text {std }}$ is monotone Lagrangian for both $\omega_{\text {std }}$ and $\phi^{*} \omega$ : For $\omega_{\text {std }}$ this is clear, and for $\phi^{*} \omega$ it follows from $\phi\left(L_{\text {std }}\right)=L_{\text {std }}$ and monotonicity of $L_{\text {std }}$ for $\omega$. Hence $L_{\text {std }}$ is monotone Lagrangian for $\omega_{t}$ for all $t$. Next, since $\phi$ preserves fibres as well as the sections $S_{0}, S_{\infty}$, the form $\phi^{*} \omega$ and thus also the form $\omega_{t}$ is cohomologous to $\omega_{\text {std }}$ for all $t$. Finally, since $\phi$ preserves the sections $S_{0}, S_{\infty}$, they are symplectic for $\phi^{*} \omega$ as well as $\omega_{\text {std }}$, hence for all $\omega_{t}$.

The desired homotopy $\omega_{t}$ is the concatenation of the homotopies constructed in the two steps. This concludes the proof of Proposition 4.9.

Remark 4.10. The two steps in the proof of Proposition 4.9 could have been performed in the opposite order: First homotope $\omega$ to the form $\phi_{*} \omega_{\text {std }}$ which has trivial holonomy along all closed curves in the base, and then homotope $\phi_{*} \omega_{\text {std }}$ to $\omega_{\text {std }}$. The latter is then a special case of the more general fact that 
two symplectic fibrations with conjugate holonomy (e.g., both having trivial holonomy) are diffeomorphic.

\subsection{Proof of the main theorem and some consequences}

We summarize the results of this and the previous section in

Theorem 4.11 (Classification of relative symplectic fibrations). Every relative symplectic fibration $\mathfrak{S}=\left(\mathcal{F}, \omega, L, \Sigma, \Sigma^{\prime}\right)$ on $S^{2} \times S^{2}$ is deformation equivalent to $\mathfrak{S}_{\text {std }}=\left(\mathcal{F}_{\text {std }}, \omega_{\text {std }}, L_{\text {std }}, S_{0}, S_{\infty}\right)$.

Proof. By Proposition $3.2, \mathfrak{S}$ is diffeomorphic to a relative symplectic fibration of the form $\widetilde{\mathfrak{S}}=\left(\mathcal{F}_{\text {std }}, \widetilde{\omega}, L_{\text {std }}, S_{0}, S_{\infty}\right)$ for some symplectic form $\widetilde{\omega}$. Combining Proposition 3.11 Proposition 4.6 and Proposition 4.9. we find a homotopy from $\widetilde{\mathfrak{S}}$ to $\mathfrak{S}_{\text {std }}$.

The main theorem will be a consequence of Theorem 4.11 and the following theorem of Gromov.

Theorem 4.12 (Gromov [10]). Let $\phi \in \operatorname{Symp}\left(S^{2} \times S^{2}, \omega_{\text {std }}\right)$ act trivially on homology. Then there exists a symplectic isotopy $\phi_{t} \in \operatorname{Symp}\left(S^{2} \times S^{2}, \omega_{\mathrm{std}}\right)$ with $\phi_{0}=\mathrm{id}$ and $\phi_{1}=\phi$.

A first consequence is

Corollary 4.13. Let $\mathfrak{S}=\left(\mathcal{F}, \omega_{\text {std }}, L, \Sigma, \Sigma^{\prime}\right)$ be a relative symplectic fibration of $M=S^{2} \times S^{2}$, where $\omega_{\text {std }}$ is the standard symplectic form. Then there exists a homotopy of relative symplectic fibrations $\mathfrak{S}_{t}=\left(\mathcal{F}_{t}, \omega_{\mathrm{std}}, L_{t}, \Sigma_{t}, \Sigma_{t}^{\prime}\right)$ with fixed symplectic form $\omega_{\text {std }}$ such that $\mathfrak{S}_{0}=\mathfrak{S}$ and $\mathfrak{S}_{1}=\left(\mathcal{F}_{\text {std }}, \omega_{\text {std }}, L_{\text {std }}, S_{0}, S_{\infty}\right)$.

Proof. By Theorem 4.11 there exists a homotopy of relative symplectic fibrations $\mathfrak{S}_{t}=\left(\mathcal{F}_{t}, \omega_{t}, L_{t}, \Sigma_{t}, \Sigma_{t}^{\prime}\right)$ with $\mathfrak{S}_{1}=\left(\mathcal{F}_{\text {std }}, \omega_{\text {std }}, L_{\text {std }}, S_{0}, S_{\infty}\right)$ and a diffeomorphism $\phi$ of $S^{2} \times S^{2}$ acting trivially on homology such that $\phi(\mathfrak{S})=\mathfrak{S}_{0}$. After applying Proposition 3.5 and modifying $\phi$ accordingly (keeping the same notation), we may assume that $\omega_{t}=\omega_{\text {std }}$ for all $t \in[0,1]$. Then $\phi$ is a symplectomorphism with respect to $\omega_{\text {std }}$, so by Gromov's Theorem 4.12 it can be connected to the identity by a family of symplectomorphisms $\phi_{t}$. Now the concatenation of the homotopies $\phi_{t}(\mathfrak{S})_{t \in[0,1]}$, and $\left(\mathfrak{S}_{t}\right)_{t \in[0,1]}$ is the desired homotopy with fixed symplectic form $\omega_{\text {std }}$.

Proof of the Main Theorem 1.1. The hypotheses of Theorem 1.1just mean that $\mathfrak{S}=\left(\mathcal{F}, \omega_{\text {std }}, L, \Sigma, \Sigma^{\prime}\right)$ is a relative symplectic fibration. By Corollary 4.13 , $\mathfrak{S}$ can be connected to $\left(\mathcal{F}_{\text {std }}, \omega_{\text {std }}, L_{\text {std }}, S_{0}, S_{\infty}\right)$ by a homotopy of relative symplectic fibrations $\mathfrak{S}_{t}=\left(\mathcal{F}_{t}, \omega_{\text {std }}, L_{t}, \Sigma_{t}, \Sigma_{t}^{\prime}\right)$ with fixed symplectic form $\omega_{\text {std }}$. In particular, $L_{t}$ is an isotopy of monotone Lagrangian tori (with respect to $\omega_{\text {std }}$ ) from $L_{0}=L$ to $L_{1}=L_{\text {std }}$. By Banyaga's isotopy extension theorem, there exists a symplectic isotopy $\phi_{t}$ with $\phi_{0}=\mathrm{id}$ and $\phi_{t}(L)=L_{t}$ for all $t$ (see the proof 
of Proposition 3.5 for the argument, ignoring the symplectic sections). Since $M$ is simply connected, the symplectic isotopy $\phi_{t}$ is actually Hamiltonian.

Another consequence of Theorem 4.11 is the following result concerning standardisation by diffeomorphisms.

Corollary 4.14 (Fixing the symplectic form). Let $\mathfrak{S}=\left(\mathcal{F}, \omega, L, \Sigma, \Sigma^{\prime}\right)$ be a relative symplectic fibration of $M=S^{2} \times S^{2}$. Then there exists a diffeomorphism $\phi$ of $S^{2} \times S^{2}$ acting trivially on homology such that $\phi^{-1}(\mathfrak{S})=\left(\widetilde{\mathcal{F}}, \omega_{\text {std }}, L_{\text {std }}, S_{0}, S_{\infty}\right)$ for some foliation $\widetilde{\mathcal{F}}$.

Proof. By Theorem 4.11, there exists a homotopy of relative symplectic fibrations $\mathfrak{S}_{t}=\left(\mathcal{F}_{t}, \omega_{t}, L_{t}, \Sigma_{t}, \Sigma_{t}^{\prime}\right)$ with $\mathfrak{S}_{1}=\left(\mathcal{F}_{\text {std }}, \omega_{\text {std }}, L_{\text {std }}, S_{0}, S_{\infty}\right)$ and a diffeomorphism $\phi$ of $S^{2} \times S^{2}$ acting trivially on homology such that $\phi(\mathfrak{S})=\mathfrak{S}_{0}$. After applying Proposition 3.5 and modifying $\phi$ accordingly (keeping the same notation), we may assume that $\left(\omega_{t}, L_{t}, \Sigma_{t}, \Sigma_{t}^{\prime}\right)=\left(\omega_{\text {std }}, L_{\text {std }}, S_{0}, S_{\infty}\right)$ for all $t \in[0,1]$. Then $\phi$ maps $\mathfrak{S}$ to $\left(\mathcal{F}_{0}, \omega_{\text {std }}, L_{\text {std }}, S_{0}, S_{\infty}\right)$.

In particular, Corollary 4.14 implies that every symplectic form $\omega$ on $S^{2} \times S^{2}$ which is compatible with a relative symplectic fibration can be pulled back to $\omega_{\text {std }}$ by a diffeomorphism $\phi \in \operatorname{Diff}_{\text {id }}(M)$. This is a special case of the deep result by Lalonde and McDuff [14] that every symplectic form $\omega$ on $S^{2} \times S^{2}$ which is cohomologous to the standard form $\omega_{\text {std }}$ can be pulled back to $\omega_{\text {std }}$ by a diffeomorphism $\phi \in \operatorname{Diff}_{\text {id }}(M)$. In fact, the hard part of the proof in [14] (using Taubes' correspondence between Seiberg-Witten and Gromov invariants) consists in showing that any such $\omega$ is compatible with a symplectic fibration with a section.

\section{A Homotopy groups of some diffeomorphism groups}

In this appendix we collect some well-known facts about the diffeomorphism and symplectomorphism groups of the disk and annulus. We fix numbers $0<a<b$ and let

$$
D:=\{z \in \mathbb{C}|| z \mid \leq b\}, \quad A:=\{z \in \mathbb{C}|a \leq| z \mid \leq b\}
$$

be equipped with the standard symplectic form $\sigma_{\text {std }}=\frac{r}{\pi\left(1+r^{2}\right)^{2}} d r \wedge d \theta$ in polar coordinates on $\mathbb{C}$ (the precise choice of the symplectic form does not matter because they are all isomorphic up to scaling by Moser's theorem). We define the following diffeomorphism groups, all equipped with the $C^{\infty}$ topology:

- $\operatorname{Diff}(D, \partial D)$ the group of diffeomorphisms of the closed disk $D$ that are equal to the identity in some neighbourhood of the boundary;

- $\operatorname{Diff}(A, \partial A)$ the group of diffeomorphisms of the closed annulus $A$ that are equal to the identity in some neighbourhood of the boundary; 
- $\operatorname{Symp}\left(A, \partial A, \sigma_{\text {std }}\right) \subset \operatorname{Diff}(A, \partial A)$ the subgroup of symplectomorphisms of $\left(A, \sigma_{\mathrm{std}}\right)$

- $\operatorname{Symp}_{0}\left(A, \partial A, \sigma_{\text {std }}\right)$ the identity component of $\operatorname{Symp}\left(A, \partial A, \sigma_{\text {std }}\right)$.

Diffeomorphisms. All results in this appendix are based on the following fundamental theorem of Smale.

Theorem A.1 (Smale [17]). The group $\operatorname{Diff}(D, \partial D)$ is contractible.

With a nondecreasing cutoff function $\rho: \mathbb{R} \rightarrow[0,1]$ which equals 0 near $(-\infty, a]$ and 1 near $[b, \infty)$, we define the Dehn twist

$$
\phi^{D}: A \rightarrow A, \quad r e^{i \theta} \mapsto r e^{i(\theta+2 \pi \rho(r))} .
$$

Corollary A.2. All homotopy groups $\pi_{i} \operatorname{Diff}(A, \partial A)$ vanish except for the group $\pi_{0} \operatorname{Diff}(A, \partial A)=\mathbb{Z}$, which is generated by the Dehn twist $\phi^{D}$.

Proof. Restriction of elements in $\operatorname{Diff}(D, \partial D)$ to the smaller disk $D_{a} \subset D$ of radius $a$ yields a Serre fibration

$$
\operatorname{Diff}(A, \partial A) \rightarrow \operatorname{Diff}(D, \partial D) \rightarrow \operatorname{Diff}^{+}\left(D_{a}\right),
$$

where Diff ${ }^{+}$denotes the orientation preserving diffeomorphisms. In view of Smale's Theorem A.1 the long exact sequence of this fibration yields isomorphisms $\pi_{i} \operatorname{Difff}^{+}\left(D_{a}\right) \cong \pi_{i-1} \operatorname{Diff}(A, \partial A)$ for all $i \geq 1$. Again by Theorem A.1 the long exact sequence of the pair $\left(\operatorname{Diff}^{+}\left(D_{a}\right)\right.$, Diff $\left.{ }^{+}\left(\partial D_{a}\right)\right)$ yields isomorphisms $\pi_{i} \operatorname{Diff}^{+}\left(\partial D_{a}\right) \cong \pi_{i} \operatorname{Diff}^{+}\left(D_{a}\right)$ for all $i$. Since $\pi_{i} \operatorname{Diff}^{+}\left(\partial D_{a}\right) \cong \pi_{i} \operatorname{Diff}^{+}\left(S^{1}\right)$ equals $\mathbb{Z}$ for $i=1$ and 0 otherwise, this proves the corollary.

For the following slight refinement of Corollary A.2 let $E \subset A$ be a circle $\{c\} \times S^{1}$ for some $c \in(a, b)$.

Corollary A.3. Every smooth loop $\left(\phi_{t}\right)_{t \in[0,1]}$ in $\operatorname{Diff}(A, \partial A)$ with $\phi_{0}=\phi_{1}=$ id and $\phi_{t}(E)=E$ for all $t$ can be contracted by a smooth family $\phi_{t}^{s} \in \operatorname{Diff}(A, \partial A)$, $s, t \in[0,1]$, satisfying $\phi_{t}^{0}=\phi_{0}^{s}=\phi_{1}^{s}=\mathrm{id}, \phi_{t}^{1}=\phi_{t}$ and $\phi_{t}^{s}(E)=E$ for all $s, t$.

Proof. For a point $e \in S^{1}$ the family of arcs $\phi_{t}([a, c] \times\{e\})$ starts and ends at $t=0,1$ with the arc $[a, c] \times\{e\}$. This shows that the loop $t \mapsto \phi_{t}(c, e)$ in $E$ is contractible, hence so is the loop $\left.\phi_{t}\right|_{E}$ in $\operatorname{Diff}(E)$. Thus we can find a family $\phi_{t}^{s} \in \operatorname{Diff}(A, \partial A), s, t \in[1 / 2,1]$, satisfying $\phi_{0}^{s}=\phi_{1}^{s}=\mathrm{id}, \phi_{t}^{1}=\phi_{t}$, $\phi_{t}^{s}(E)=E$ for all $s, t$, and $\left.\phi_{t}^{1 / 2}\right|_{E}=$ id for all $t$. Now apply Corollary A.2 to contract the loops $\left.\phi_{t}^{1 / 2}\right|_{[a, c] \times S^{1}}$ in $\operatorname{Diff}\left([a, c] \times S^{1}, \partial[a, c] \times S^{1}\right)$ and $\left.\phi_{t}^{1 / 2}\right|_{[c, b] \times S^{1}}$ in $\operatorname{Diff}\left([c, b] \times S^{1}, \partial[c, b] \times S^{1}\right)$.

Symplectomorphisms. The following result is an immediate consequence of Corollary A.2 and Moser's theorem. 
Proposition A.4. The groups $\operatorname{Diff}(A, \partial A)$ and $\operatorname{Symp}\left(A, \partial A, \sigma_{\text {std }}\right)$ are weakly homotopy equivalent. In particular, $\operatorname{Symp}_{0}\left(A, \partial A, \sigma_{\mathrm{std}}\right)$ is contractible.

Remark A.5. The Dehn twist $\phi^{D}$ defines an element in $\operatorname{Symp}\left(A, \partial A, \sigma_{\text {std }}\right)$, which according to Proposition A.4 generates $\pi_{0} \operatorname{Symp}\left(A, \partial A, \sigma_{\text {std }}\right)$.

Finally, we need the following refinement of Proposition A.4 Again, let $E \subset A$ be a circle $\{c\} \times S^{1}$ for some $c \in(a, b)$.

Lemma A.6. Each $\phi \in \operatorname{Symp}_{0}\left(A, \partial A, \sigma_{\text {std }}\right)$ with $\phi(E)=E$ can be connected to the identity by a smooth path $\phi_{t} \in \operatorname{Symp}_{0}\left(A, \partial A, \sigma_{\text {std }}\right)$ satisfying $\phi_{t}(E)=E$ for all $t \in[0,1]$.

Proof. After applying Moser's theorem and changing the values of $a, b, c$ (viewing $A$ as a cylinder), we may assume that $\sigma_{\text {std }}=d r \wedge d \theta$ and $c=0$. We connect $\phi$ to the identity in 4 steps.

Step 1. The restriction $f(\theta):=\phi(0, \theta)$ of $\phi$ to $E$ defines an element in the group $\operatorname{Diff}^{+}\left(S^{1}\right)$ of orientation preserving diffeomorphisms of the circle. Since this group is path connected, there exists a smooth family $f_{t} \in \operatorname{Diff}^{+}\left(S^{1}\right)$ with $f_{0}=$ id and $f_{1}=f$. This family if generated by the time-dependent vector field $\xi_{t}$ on the circle defined by $\xi_{t}\left(f_{t}(\theta)\right):=\dot{f}_{t}(\theta)$. Let $H_{t}: A \rightarrow \mathbb{R}$ be a smooth family of functions satisfying $H_{t}(r, \theta)=-r \xi_{t}(\theta)$ near $E$ and $H_{t}=0$ near $\partial A$. A short computation shows that the Hamiltonian vector field of $H_{t}$ agrees with $\xi_{t}$ on $E$. It follows that the Hamiltonian flow $\psi_{t}$ of $H_{t}$ satisfies $\left.\psi_{t}\right|_{E}=f_{t}$, in particular $\left.\psi_{1}\right|_{E}=f_{1}=\left.\phi\right|_{E}$. Thus $\phi_{t}:=\phi \circ \psi_{t}^{-1}$ is a smooth path in $\operatorname{Symp}_{0}\left(A, \partial A, \sigma_{\text {std }}\right)$ with $\phi_{t}(E)=E$ connecting $\phi$ to $\phi_{1}$ satisfying $\left.\phi_{1}\right|_{E}=$ id. After renaming $\phi_{1}$ back to $\phi$, we may hence assume that $\left.\phi\right|_{E}=$ id.

Step 2. Let us write $\phi(r, \theta)=(P(r, \theta), Q(r, \theta)) \in \mathbb{R} \times S^{1}$. Since $\left.\phi\right|_{E}=$ id and $\phi$ is symplectic, the functions $P, Q$ satisfy

$$
P(0, \theta)=0, \quad Q(0, \theta)=\theta, \quad \frac{\partial P}{\partial r}(0, \theta)=1 .
$$

For $s \in(0,1]$ consider the dilations $\tau_{s}(r, \theta):=(s r, \theta)$ on $A$. Since $\tau_{s}^{*}(d r \wedge d \theta)=$ $s d r \wedge d \theta$, the maps $\psi_{s}:=\tau_{s}^{-1} \circ \phi \circ \tau_{s}$ are symplectic. Since

$$
\psi_{s}(r, \theta)=\left(\frac{1}{s} P(s r, \theta), Q(s r, \theta)\right) \underset{s \rightarrow 0}{\longrightarrow}\left(r \frac{\partial P}{\partial r}(0, \theta), Q(0, \theta)\right)=(r, \theta),
$$

the family $\psi_{s}$ extends smoothly to $s=0$ by the identity (this is a fibered version of the Alexander trick). It follows that for a sufficiently small $\varepsilon>0$ we have a smooth family of symplectic embeddings $\psi_{s}: A_{\varepsilon}:=[-\varepsilon, \varepsilon] \times S^{1} \hookrightarrow A$, $s \in[0,1]$, with $\psi_{s}(E)=E, \psi_{0}=\mathrm{id}$, and $\psi_{1}=\phi$. We extend this family to smooth diffeomorphisms $\widetilde{\psi}_{s}: A \rightarrow A$ with $\widetilde{\psi}_{s}=$ id near $\partial A$ and $\widetilde{\psi}_{1}=\phi$. Since $\widetilde{\psi}_{s}$ preserves the annuli $A^{-}:=[a, 0] \times S^{1}$ and $A^{+}:=[0, b] \times S^{1}$, it satisfies $\int_{A^{ \pm}} \widetilde{\psi}_{s}^{*} \sigma_{\mathrm{std}}=\int_{A^{ \pm}} \sigma_{\mathrm{std}}$ for all $s \in[0,1]$. By Banyaga's Theorem 3.4 applied to the isotopy $t \mapsto \phi^{-1} \circ \widetilde{\psi}_{1-t}$ and the set $X:=[a, a+\varepsilon] \times S^{1} \cup[b-\varepsilon, b] \times S^{1} \cup A_{\varepsilon}$ 
for some possibly smaller $\varepsilon>0$, there exists a symplectic isotopy $\phi_{s}: A \rightarrow A$, $s \in[0,1]$, with $\phi_{1}=\phi$ and $\left.\phi_{s}\right|_{X}=\left.\widetilde{\psi}_{s}\right|_{X}$. In particular, $\phi_{s} \in \operatorname{Symp}_{0}\left(A, \partial A, \sigma_{\text {std }}\right)$ preserves $E$ and $\left.\phi_{0}\right|_{A_{\varepsilon}}=$ id. After renaming $\phi_{0}$ back to $\phi$, we may hence assume that $\phi=$ id on an annulus $A_{\varepsilon}$ around $E$.

Step 3. Since $\left.\phi\right|_{A_{\varepsilon}}=$ id, it restricts to maps $\left.\phi\right|_{A^{ \pm}} \in \operatorname{Symp}\left(A^{ \pm}, \partial A^{ \pm}, \sigma_{\text {std }}\right)$. By Proposition A.4 and Remark A.5, $\left.\phi\right|_{A^{ \pm}}$can be connected in $\operatorname{Symp}\left(A^{ \pm}, \partial A^{ \pm}, \sigma_{\text {std }}\right)$ to a multiple $\left(\phi_{ \pm}^{D}\right)^{k_{ \pm}}$of the Dehn twist on $A^{ \pm}$. Since $\phi$ belongs to the identity component $\operatorname{Symp}_{0}\left(A, \partial A, \sigma_{\text {std }}\right)$, it follows that $k_{+}=-k_{-}$. Hence we can simultaneously unwind the Dehn twists to connect the map $\psi$ which equals $\left(\phi_{ \pm}^{D}\right)^{k_{ \pm}}$on $A^{ \pm}$to the identity by a path $\psi_{t}$ in $\operatorname{Symp}_{0}\left(A, \partial A, \sigma_{\text {std }}\right)$ fixing $E$ (but not restricting to the identity on $E)$. Thus $\phi_{t}:=\phi \circ \psi_{t}^{-1}$ is a path in $\operatorname{Symp}_{0}\left(A, \partial A, \sigma_{\text {std }}\right)$ with $\phi_{t}(E)=E$ connecting $\phi$ to $\phi_{1}$ such that $\left.\phi_{1}\right|_{A^{ \pm}}$belongs to the identity component $\operatorname{Symp}_{0}\left(A^{ \pm}, \partial A^{ \pm}, \sigma_{\text {std }}\right)$. Again, we rename $\phi_{1}$ back to $\phi$.

Step 4. Finally, we apply Proposition A.4 on $A^{ \pm}$to connect $\left.\phi\right|_{A^{ \pm}}$to the identity by a path $\phi_{t}^{ \pm}$in $\operatorname{Symp}_{0}\left(A^{ \pm}, \partial A^{ \pm}\right)$. The maps $\phi_{t}^{ \pm}$fit together to a path $\phi_{t} \in$ $\operatorname{Symp}_{0}\left(A, \partial A, \sigma_{\text {std }}\right)$ fixing $E$ that connects $\phi$ to the identity.

\section{References}

[1] P. Albers and U. Frauenfelder, A non-displaceable Lagrangian torus in $T^{*} S^{2}$, Comm. Pure Appl. Math. 61, 1046-1051 (2008).

[2] A. Banyaga, Sur la structure du groupe des difféomorphismes qui préservent une forme symplectique, Comment. Math. Helv. 53, no. 2, 174-227 (1978).

[3] P. Biran and O. Cornea, Rigidity and uniruling for Lagrangian submanifolds, Geom. Topol. 13, no. 5, 2881-2989 (2009).

[4] Y. Chekanov and F. Schlenk, Notes on monotone Lagrangian twist tori, Electron. Res. Announc. Math. Sci. 17, 104-121 (2010).

[5] Y. Eliashberg and L. Polterovich, The problem of Lagrangian knots in four-manifolds, Geometric topology (Athens, GA, 1993), 313-327, AMS/IP Stud. Adv. Math., Vol. 2.1 (1997).

[6] M. Entov and L. Polterovich, Rigid subsets of symplectic manifolds, Compos. Math. 145, 773-826 (2009).

[7] J. Evans, Lagrangian spheres in Del Pezzo surfaces, Journal of Topology 3 (1), 181-227 (2010).

[8] K. Fukaya, Y.-G. Oh, H. Ohta and K. Ono, Lagrangian intersection Floer theory: anomaly and obstruction. Part II, AMS/IP Studies in Advanced Mathematics, Vol. 46.2 (2009). 
[9] A. Gadbled, On exotic monotone Lagrangian tori in $\mathbb{C} P^{2}$ and $S^{2} \times S^{2}$, J. Symp. Geom. 11, no. 3, 343-361 (2013).

[10] M. Gromov, Pseudo holomorphic curves in symplectic manifolds, Invent. math. 82, 307-347 (1985).

[11] R. Hind, Lagrangian spheres in $S^{2} \times S^{2}$, Geom. Funct. Analysis (2) 14, 303-318 (2004).

[12] R. Hind, Lagrangian unknottedness in Stein surfaces, Asian J. Math. 16, no. 1, 1-36 (2012).

[13] A. Ivrii, Lagrangian unknottedness of tori in certain symplectic 4manifolds, PhD thesis, Stanford University (2003).

[14] F. Lalonde and D. McDuff, The classification of ruled symplectic 4manifolds, Math. Res. Letters 3, 769-778 (1996).

[15] D. McDuff and D. Salamon, Introduction to Symplectic Topology, Oxford University Press, 2nd edition (2005).

[16] J. Oakley and M. Usher, On Certain Lagrangian Submanifolds of $S^{2} \times S^{2}$ and $C P^{n}, \operatorname{arXiv:1311.5152}$

[17] S. Smale, Diffeomorphisms of the 2-sphere, Proc. Amer. Math. Soc. 10, 621-626 (1959).

[18] M. Schwingenheuer, Hamiltonian unknottedness of certain monotone Lagrangian tori in $S^{2} \times S^{2}, \mathrm{PhD}$ thesis, Ludwig-MaximiliansUniversität München (2010).

[19] R. Vianna, On exotic Lagrangian tori in $\mathbb{C} P^{2}$, Geom. Topol. 18, no. 4, 2419-2476 (2014).

[20] R. Vianna, Infinitely many exotic monotone Lagrangian tori in $\mathbb{C} P^{2}$, arXiv:1409.2850. 